LA W RENCE LIVERMORE N A TIO NAL LABORATORY

\title{
Final Report: "Sensitivity Study of LIFE Chamber Dynamics"
}

C. Pantano-Rubino, H. Uddin, R. Kramer

October 29, 2013 
This document was prepared as an account of work sponsored by an agency of the United States government. Neither the United States government nor Lawrence Livermore National Security, LLC, nor any of their employees makes any warranty, expressed or implied, or assumes any legal liability or responsibility for the accuracy, completeness, or usefulness of any information, apparatus, product, or process disclosed, or represents that its use would not infringe privately owned rights. Reference herein to any specific commercial product, process, or service by trade name, trademark, manufacturer, or otherwise does not necessarily constitute or imply its endorsement, recommendation, or favoring by the United States government or Lawrence Livermore National Security, LLC. The views and opinions of authors expressed herein do not necessarily state or reflect those of the United States government or Lawrence Livermore National Security, LLC, and shall not be used for advertising or product endorsement purposes.

This work performed under the auspices of the U.S. Department of Energy by Lawrence Livermore National Laboratory under Contract DE-AC52-07NA27344. 


\title{
$\underline{\mathbb{I}}$ I I L L I N N O I S
}

\section{Final Report: "Sensitivity Study of LIFE Chamber Dynamics"}

\author{
Principal Investigator: Carlos Pantano-Rubino* \\ Other Personnel: Hasib Uddin (graduate assistant), Richard Kramer (post-doc) ${ }^{\dagger}$ \\ Department of Mechanical Science and Engineering \\ University of Illinois at Urbana-Champaign
}

November 5, 2013

*Contact: e-mail: cpantano@illinois.edu, phone: 217-244-1412

${ }^{\dagger}$ Currently at Sandia National Laboratory 


\section{Preface}

The objective of this research is to investigate the turbulent flow state in a prototype of the LIFE fusion chamber, over many fusion cycles, using high-fidelity three-dimensional computational modeling. A fusion cycle duration is defined here as the time between laser shots (that induce fusion of the fuel stored in the capsule). The main problem of interest discussed in this report is the study of the sensitivity of the flow conditions in the fusion chamber to the radiation parameters used in the BUCKY code (Univ. Wisconsin Madison) that give the initial conditions for the present simulations. Of particular interest is the asymptotic " ${ }^{t h}$-state" cycle in the model fusion chamber and vacuum vessel assembly. The asymptotic (but unsteady) state was reached by advancing in time an initial value problem with estimated initial data that are thought to be representative of the fusion chamber state (in the mean). These conditions were provided to the Illinois team by LLNL. Many details of the flow solver, strategy and other auxiliary information can be found in a previous report entitled "CFD Simulation in Support of the Dynamic Chamber Systems Strategic Initiative." Here, several quantities were investigated in detail. First, the global balance of mass in the fusion chamber and vacuum vessel. Second, the state of the density field inside the chamber: turbulence and density spectra, mean and fluctuation of the density. Third, to develop an understanding of the effect of forced recirculation imposed on the chamber-vacuum vessel ensemble, to understand the behavior and distribution of the capsule debris throughout the domain and instantaneous fluxes through the numerous ports and openings of the chamber. Fourth, and finally, to elucidate transport properties near the relatively cold walls of the chamber that might affect the chemistry of the complex fluid mixture from calculated temperature condition on the walls. These four questions have been investigated with different degrees of fidelity (as detailed below) assuming as few simplifications as possible while maintaining many elements of the complex geometry of the fusion chamber. Finally, the simulations do not include the effects of radiation explicitly, which is expected to be critical during the instants immediately after a laser pulse and fusion ignition, and instead a rational "handshake" procedure was developed to derive initial conditions consistent with those produced by BUCKY under different assumptions. 


\section{Contents}

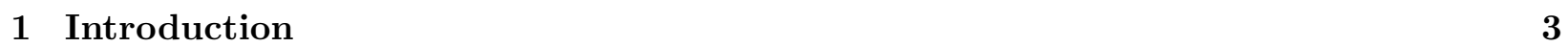

2 Handshake procedure - BUCKY to VTF 5

2.1 Energy source specification $\ldots \ldots \ldots \ldots \ldots \ldots \ldots$

2.2 Handshake model $\ldots \ldots \ldots \ldots \ldots \ldots \ldots \ldots \ldots \ldots \ldots$

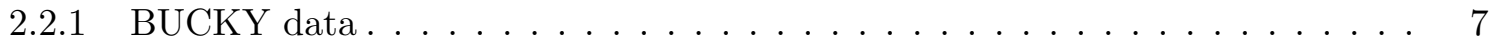

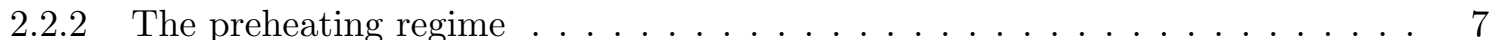

2.2 .3 Calculation of the blast energy $\ldots \ldots \ldots \ldots \ldots \ldots$

2.2 .4 Redistribution of the mass and energy . . . . . . . . . . . . . . . . . . 8

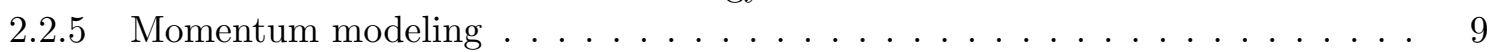

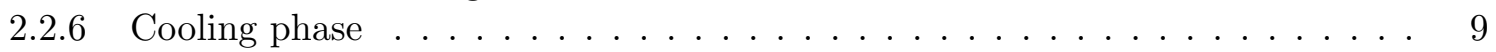

2.3 Multishot 1D Simulation Test $\ldots \ldots \ldots \ldots \ldots \ldots \ldots$

3 Simulation Description and Parameters 13

$\begin{array}{lll}4 & \text { Simulation Results } & 13\end{array}$

4.1 Global behavior of the system . . . . . . . . . . . . . . . . . . . . 14

4.2 Approaching the $\mathrm{n}^{t h}$ shot state $\ldots \ldots \ldots \ldots \ldots \ldots \ldots \ldots$

4.3 Hohlraum Environment . . . . . . . . . . . . . . . . . . . . . . . . 22

4.4 Turbulence Spectra . . . . . . . . . . . . . . . . . . . . . . . . . 24

4.5 Temperature distribution on the fusion chamber walls . . . . . . . . . . . . . . . 29

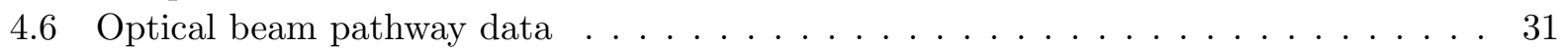

\begin{tabular}{|lll}
5 & Conclusions and Perspective for Future Improvements & 37
\end{tabular} 


\section{Introduction}

The simulations used a version of the Virtual Test Facility (VTF) developed at Caltech (by the PI and others) as part of the Accelerated Strategic Computing Initiative (ASCI) of DOE under the direction of Prof. Daniel Meiron, that is maintained at the University of Illinois at UrbanaChampaign. The suite of solvers implement the compressible Navier-Stokes equations using a largeeddy simulation (LES) turbulence modeling closure, complex geometry using an embedded level set representation, adaptive mesh refinement (AMR), and parallel supercomputing using the message passing interface (MPI). A number of enhancements with respect to the Caltech version have been used in the present effort. These include new enhanced discretization stencils for LES. In addition, a number of special features were specifically implemented in the simulations to ensure consistent forced flow recirculation in the chamber. The fusion chamber and vacuum vessel geometry is shown in figure 1. The current generation of the chamber simulation setup and associated codes include:

- A cylindrical vacuum vessel encompassing the fusion chamber. It has 48 rectangular ports at the top and bottom caps which are aligned with the fusion chamber ports as shown in figure 1.

- Recirculation flow is injected through all the vacuum vessel ports instead of a single injection port at the top; as was done in previous versions. The recirculation flux is maintained to ensure the clearing ratio of $0.83 \%$; specified by LLNL.

- A specifically-designed controller is used through the drain pipe to ensure balance of mass in the whole system. This controller prevents mass accumulation in the system by regulating the drain outlet velocity.

- An updated handshake procedure is used to approximately reconcile the more physicallyaccurate BUCKY data provided as the starting point of the sensitivity studies.

- The shot frequency is set to $16 \mathrm{~Hz}$, resulting in an intershot period of $62.5 \mathrm{~ms}$.

The fusion chamber model we employed in all the simulations has the following dimensions: $600 \mathrm{~cm}$ internal radius, $740 \mathrm{~cm}$ external radius, $140 \mathrm{~cm}$ wall thickness, 24 openings or ports on the top and bottom hemispheres (in two 12 ports groups at $25^{\circ}$ and $47.5^{\circ}$ from the vertical coordinate, respectively, which are denoted in the rest of the report as $\pm z \measuredangle 25^{\circ}$ and $\pm z \measuredangle 47.5^{\circ}$ ), a drain of internal radius $70 \mathrm{~cm}$ and external radius $210 \mathrm{~cm}$ reaching all the way to the vacuum vessel. 


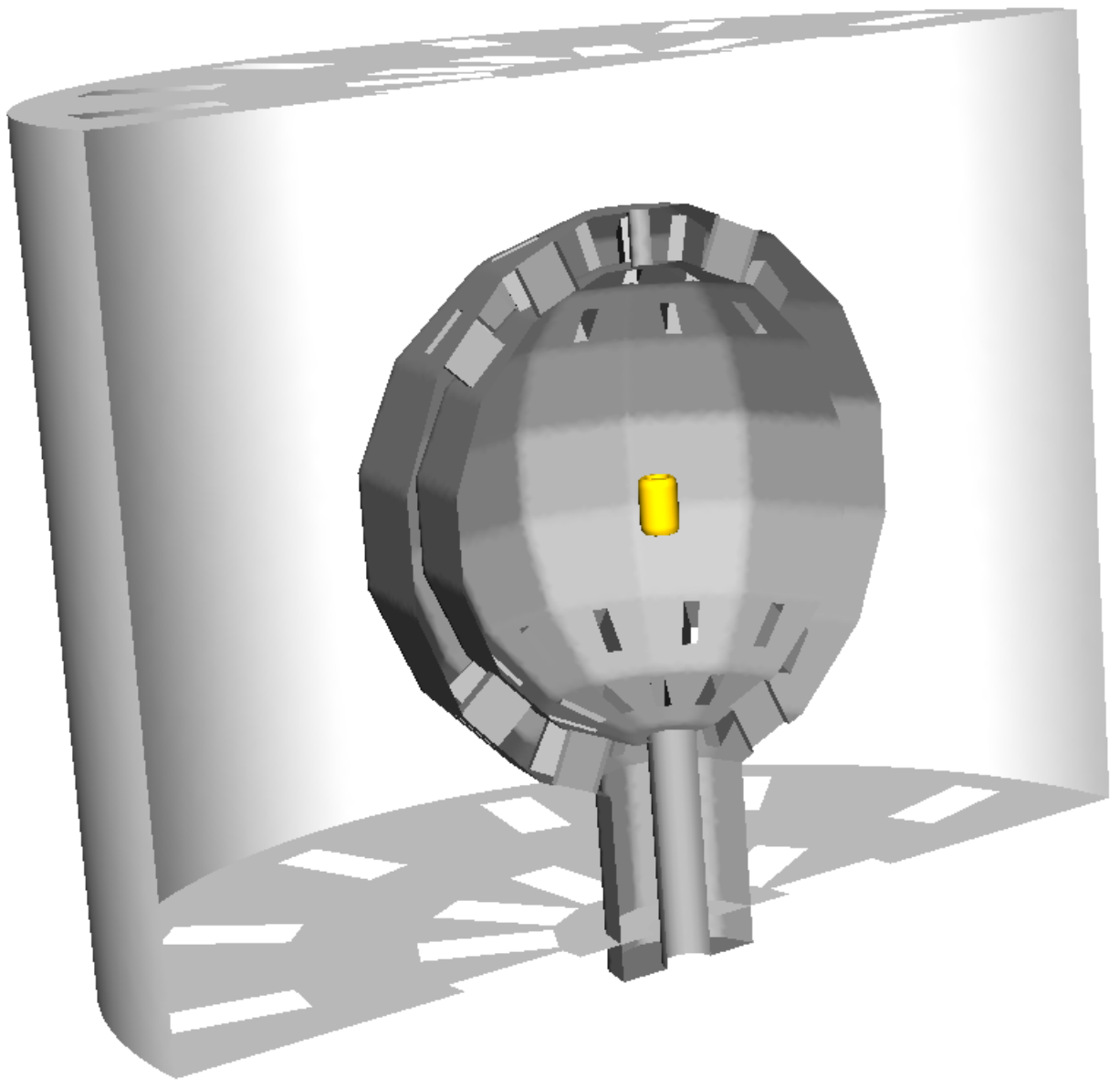

Figure 1: A cut of the geometrical configuration showing the fusion chamber inside a cylindrical vacuum vessel. The fusion target size is magnified in the center of the chamber for representation purposes only. 


\section{Handshake procedure - BUCKY to VTF}

This document is an update to previous reports ${ }^{112}$ and presumes some familiarity with those document. The following nomenclature is employed to ensure precise understanding of the concepts described below:

- Shot period: The phenomena that takes place when the lasers impact the target. This includes optical, photon, material and phase change, nuclear, and ionization processes. From a hydrodynamic point of view, this takes place over such small timescales, with respect to wave-propagation phenomena, that it could be considered an instantaneous process.

- Radiative period: In this period, the target has disintegrated, a plasma is formed and radiative processes are dominant. This period is roughly of the order of a fraction of a millisecond.

- Blast period: In this period the plasma has mostly neutralized itself and hydrodynamical phenomena are dominant. This period extends up to the time where the blast wave reaches the walls of the chamber. Based on shock theory, there are two regimes in this period. A strong blast period where the blast wave is characterized by a strong shock (according to strong shock theory), and usually corresponds to the time when the shock is about midway between the center and the walls of the chamber. Then, this is followed by a weak blast period where the blast wave is weak and blast theory is no longer applicable.

- Hydrodynamic period: includes the weak blast period up to (but just before) the next shot.

- Handshake model: A hydrodynamic model that replaces the more physically accurate solution immediately after the radiative period with a purely neutral gas (but in some sense equivalent) model approximating the conditions of the fluid.
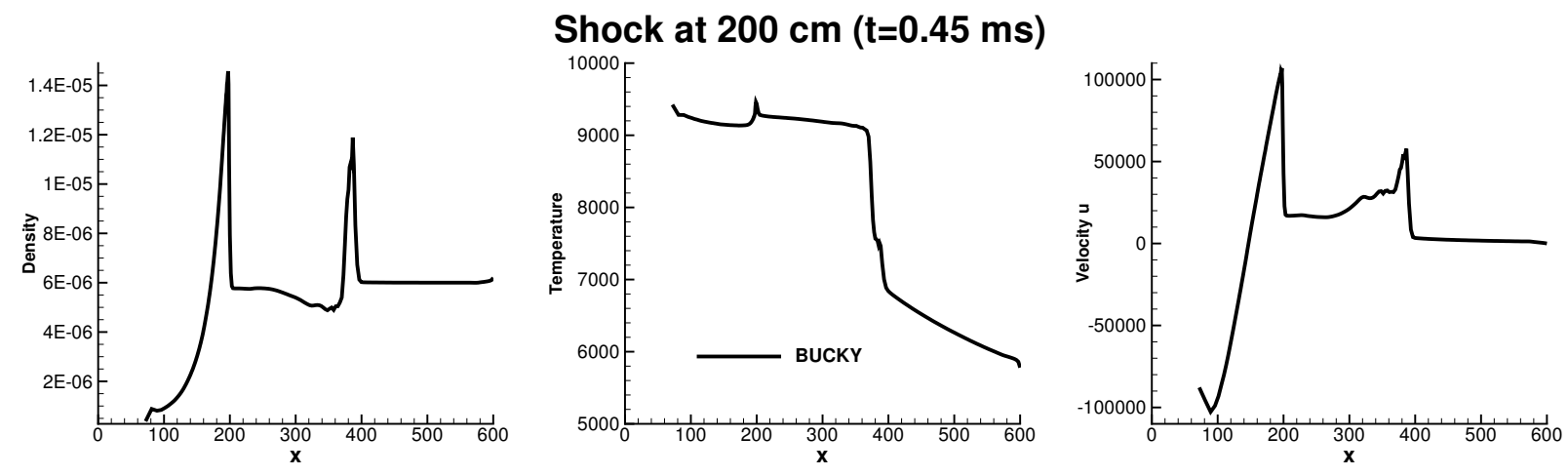

Figure 2: BUCKY results at $t=0.45 \mathrm{~ms}$.

\subsection{Energy source specification}

The detailed one-dimensional (spherical) model used by BUCKY includes radiation, plasma physics, and hydrodynamics. The low dimensionality of these simulations enable very detailed studies of the phenomena induced by the ignition source. In this model, the radiative period is modeled accurately, it includes the Marshak wave that sets the whole fluid in the chamber to approximate 1 $\mathrm{eV}$. During this period, the fluid in the center of the chamber has barely moved. Then, a blast wave 
starts to propagate radially outwards and this modifies substantially the density, momentum and energy of the Xenon in the chamber. Figure 2 shows BUCKY results at $t=0.45 \mathrm{~ms}$ (end of radiative period and beginning of blast period) where the main blast wave has a radius of approximately 200 $\mathrm{cm}$ (a second discontinuity is observed at around $R_{C}=370 \mathrm{~m}$ but its speed is much smaller than that of the main blast wave). In this simulation, the flow state ahead of the blast is not uniform due to radiative phenomena (the radial velocity is not zero, presumably because of the expansion induced by the heating of the fluid during the radiation period).

The following complications must be taken into account when developing a handshake model to be used in the complex flow and geometry of the chamber hydrodynamic studies. First, the model must have an additive structure. Specifically, the model must be incorporated as an additive source of mass, momentum, and energy to the current state of the chamber. This is due to the fact that the chamber fluid does not revert back to uniform conditions at the end of the hydrodynamic period. Second, one must account for the added mass and momentum of the target (that is disintegrated during the laser ignition process). This mass and momentum must be added to the current nonuniform fluid in the center of the chamber. Third, and finally, the radiative period processes, including the passage of the Marshak wave, must be incorporated in some fashion to set the conditions ahead of the blast wave at appropriate values.

The natural simplification of the complex energy source is to replace the more realistic flow state at the end of the radiation period with that of an instantaneous energy deposition in the center of the domain using the similarity theory of Taylor and Sedov. Under this assumption, strong shock theory and self-similarity can be used to obtain semi-analytical solutions of the compressible Euler equations. The procedure starts by observing, from dimensional arguments, that the radius of the blast must obey

$$
R(t)=k\left(\frac{E_{o}}{\rho_{o}}\right)^{1 / 5} t^{2 / 5},
$$

where $E_{o}$ is the deposited energy, $\rho_{o}$ is the density of the gas in the unperturbed region, and $k$ is a constant of integration. This allows the definition of a self-similar coordinate $\xi=r / R(t)$ that introduced into the conservation equations for mass, momentum, and energy,

$$
\begin{aligned}
\frac{\partial \rho}{\partial t}+\frac{\partial(\rho u)}{\partial r}+2 \frac{\rho u}{r} & =0 \\
\frac{\partial u}{\partial t}+u \frac{\partial u}{\partial r}+\frac{1}{\rho} \frac{\partial p}{\partial r} & =0 \\
\frac{\partial p}{\partial t}+u \frac{\partial p}{\partial r}-a^{2}\left(\frac{\partial \rho}{\partial t}+u \frac{\partial \rho}{\partial r}\right) & =0
\end{aligned}
$$

reduce the equations to simple ordinary differential equations that can be solved with commonly available desktop software; see Whitham ${ }^{3}$ for boundary conditions and the reduced equations. In equation (4), $a$ denotes the speed of sound $\sqrt{\gamma p / \rho}$, where $\gamma$ is the specific heat ratio for Xenon. By matching the time to the radius of the shock at $t=0.45 \mathrm{~ms}$ (strong blast period) in the BUCKY simulation to the ideal blast solution, it is possible to deduce $E_{o}$ (the deposited energy), to be 0.784 MJ (at the conditions of the BUCKY simulation given to us). Attempting to match the blast solution at other times is not straightforward. Earlier times than $0.45 \mathrm{~ms}$ do not display complete neutralization of the plasma and radiation is expected to be a dominant effect (which is missing in the blast solution). Matching at latter times is also not feasible because the blast solution is valid as long as the shock is strong. In the conditions of the chamber, the blast is only strong enough during the first $1 \mathrm{~ms}$ or so (up to about $300 \mathrm{~cm}$ ). The radial expansion makes the shock very week beyond $300 \mathrm{~cm}$ and therefore one could not use the arrival time at $400 \mathrm{~cm}$ to determine 
the energy $E_{o}$. Therefore, matching with the BUCKY solution was decided to take place at $t=0.45$ $\mathrm{ms}$ (or when the shock is at $200 \mathrm{~cm}$ radius). One should keep in mind that the main goal of the chamber simulations is to estimate the hydrodynamic state of the flow in the chamber, mostly just before a new shot, but also to understand transport, dispersion and migration of the debris residue. Therefore, the radiative processes whose energy ends being adsorbed by chamber walls (the complex heat exchanger) cannot be modeled in the hydrodynamic simulations. The expectation is that the handshake model includes the bulk mass and energy deposited in the fluid and some level of momentum (velocity) produced by the early blast wave. The first and second processes can be accounted easily by distributing the mass of the capsule and the equivalent energy $E_{o}$ determined above. The momentum component is harder to include accurately because the blast wave velocity is sensitive to the conditions encountered in the chamber in the undisturbed region ahead of the wave.

\section{$2.2 \quad$ Handshake model}

We assume information from the BUCKY simulations is available and transform this data into a suitable model for the Chamber hydrodynamic simulation. We detail below every step and calculation required in Version 2 of the handshake model to implement the approximate matching of the two physical models of the process.

\subsubsection{BUCKY data}

The handshake model assumes the following information is obtained from BUCKY:

- Radius of the shock wave, $R_{B}$, at the handshake time $t_{B}$ at the end of the radiation period.

- Preheat temperature of the post Marshak wave, $T_{B}$.

- Energy loss model for high-temperature regions at the center of the chamber, $\dot{q}_{B}(T)$.

\subsubsection{The preheating regime}

While the handshake takes place at $t=t_{B}$ after the shot period, the gas in the chamber simulation is preheated to post-Marshak wave conditions before this time is reached. This is implemented in the VTF by enabling a source term in the energy equation that drives the temperature inside the chamber to $T_{B}$ for radia below $R_{C}$ (the outer gas up to the chamber walls is left unmodified). The source is of the form

$$
\dot{q}_{M}=-C_{M} R_{g} \rho_{c} \frac{\left(T-T_{B}\right)}{t_{B}}, \quad \text { for } \quad t<t_{B} \quad \& \quad r<R_{C},
$$

where $C_{M}$ is a constant, $R_{g}$ is the ideal gas constant for Xenon, and $\rho_{c}$ is the initial average density of the gas in the fusion chamber. In all simulations, $C_{M}=5$. The energy source acts as a driver that drives the temperature exponentially fast (with rate $C_{M}$ ) to the target temperature $T_{B}$ in the time $t_{B}$.

\subsubsection{Calculation of the blast energy}

The blast energy is calculated from the ideal theory, according to

$$
E_{o}=\frac{\rho_{o}}{t_{B}^{2}}\left(\frac{R_{B}}{k}\right)^{5},
$$


where $\rho_{o}$ is the density in the chamber ahead of the blast, and $k=1.03871$ for $\gamma=5 / 3$; see Whitham ${ }^{3}$. At first sight, one might think that this is sufficient to connect the BUCKY simulation with the VTF Chamber hydrodynamic model and one could use the blast (self-similar) solutions to reinitialize the center of the chamber. But, inspection of the self-similar blast solution shows that the temperature becomes singular at $r=0$. Therefore, the energy $E_{o}$ must be redistributed over the blast region $r<R_{B}$ to prevent the temperature from becoming singular (or too high in the simulation that uses a discrete grid).

\subsubsection{Redistribution of the mass and energy}

Since $E_{o}$ cannot be deposited according to the blast solution, because it is singular at the origin, the energy is redistributed according to the following radial function

$$
f_{\alpha, \beta, \delta}(r)=\frac{\delta+\beta \exp \left(\alpha\left(r / R_{B}-1\right)\right)}{\frac{4 \pi}{3} R_{B}^{3}\left\{\delta+3 \beta\left[\alpha(\alpha-2)+2-2 e^{-\alpha}\right] \alpha^{-3}\right\}},
$$

that one can verify integrates to unity on the volume $r \leq R_{B}$ for all $\alpha, \beta$, and $\delta$. The new internal energy per unit volume of the fluid immediately after the handshake time is given by

$$
(E)_{t_{B}^{+}}=(E)_{t_{B}^{-}}+E_{o} f_{\alpha, \beta, \delta}(r)
$$

where $\alpha=2, \beta=1$, and $\delta=1, E=p /(\gamma-1)$. The quanties $(E)_{t_{B}^{-}}$and $(E)_{t_{B}^{+}}$denote the value of $E$ before and after handshake, respectively.

Similarly, one cannot specify the density according to the ideal blast solution because it becomes very small (near vacuum) at the center of the chamber. But, differently from the energy, mass is conserved and there is not net addition of mass in a blast. One can verify that the total mass before the instantaneous addition of energy (at $t=0$ ) in the region $r<R_{B}$ is identical to the mass behind the shock in the self-similar solution at $t=t_{B}$ (this works at all times, not just at handshake time). Therefore, we redistribute the mass by first calculating the mass in the center of the chamber, obtained from the integral

$$
M_{o}=\iiint_{r<R_{B}}(\rho)_{t_{B}^{-}} d x d y d z
$$

and replace the density in this region by

$$
(\rho)_{t_{B}^{+}}=\left(M_{o}+M_{T}\right) f_{\alpha, \beta, \delta}(r),
$$

with $\alpha=6, \beta=1$, and $\delta=1 / 8$, and $M_{T}$ denotes the mass of the target that was vaporized by the laser shot, which is not lost. Note that the ideal blast theory does not contemplate the addition of a finite mass initially (non-selfsimilar theory is required for this refinement). Also note that $M_{o}$ is recalculated before each handshake is introduced and depends on the current conditions of the gas in the chamber. This ensures that the total mass in the chamber is conserved and that the target mass is added to the chamber.

The values of the parameters $\alpha, \beta$, and $\delta$ for mass and energy were selected after several tests to determine how sensitive the solution was to the choice of parameters. The values above were satisfactory but are by no means uniquely defined. The only consolation is that the full gas dynamics simulations show that the shock propagation speed is not very sensitive to these values (to be shown shortly below). 


\subsubsection{Momentum modeling}

The modeling of the contribution of the blast to linear momentum in the chamber is more empirical than that of mass and energy. This is because the shock radius evolution as a function of time depends (in a non-negligible manner) on the velocity of the gas ahead of the outward-going blast wave. But, it is recognized that the velocities induced by the blast are important and contribute to the turbulence state of the chamber (this aspect of the blast physics was ignored in all our previous chamber hydrodynamic studies). The model employed in this version of the handshake uses directly the information given by BUCKY. The following linear momentum correction is 'added' to the region $r<R_{B}$,

$$
v_{r}=U_{o} \begin{cases}-2 \frac{r}{R_{B}} & r<\frac{1}{2} R_{B}, \\ -3+4 \frac{r}{R_{B}} & r>\frac{1}{2} R_{B},\end{cases}
$$

where $U_{o}$ is the peak velocity in the BUCKY simulation. The velocity $v_{r}$ is the radial component (spherically symmetric) that is projected along the corresponding Cartesian directions. Finally, the momentum modeling also includes the momentum of the target (which should also not be lost completely due to the blast), giving the following additional momentum model

$$
\begin{aligned}
(\rho u)_{t_{B}^{+}} & =(\rho u)_{t_{B}^{-}}+(\rho)_{t_{B}^{+}} v_{r}(r) \frac{x}{r} \\
(\rho v)_{t_{B}^{+}} & =(\rho v)_{t_{B}^{-}}+(\rho)_{t_{B}^{+}} v_{r}(r) \frac{y}{r} \\
(\rho w)_{t_{B}^{+}} & =(\rho w)_{t_{B}^{-}}+(\rho)_{t_{B}^{+}} v_{r}(r) \frac{z}{r}-M_{T} f_{\alpha, \beta, \delta}(r) U_{T},
\end{aligned}
$$

where $U_{T}$ denotes the velocity of the target.

\subsubsection{Cooling phase}

In the actual chamber, and after a shot takes place, there is still sufficiently high temperature in the center of the chamber that radiation towards the fusion-chamber walls cannot be neglected. This is modeled by introducing a heat sink in the chamber, of the following form

$$
\dot{q}_{S}=-C_{M} R_{g} \rho_{c} \frac{\left(T-T_{S}\right)}{t_{S}} \text { for } T>T_{S}
$$

where $C_{M}$ is the same constant as before, $T_{S}$ is the sink temperature and $t_{S}$ is the sink time scale.

\subsection{Multishot 1D Simulation Test}

An example of the application of the handshake model described above is discussed here for the simplified one-dimensional (spherically symmetric) VTF problem. This is not the Chamber VTF application but it uses the same methods and code that the fully three-dimensional code employs. Figure 3(top) shows the initial density, temperature and velocity. At the handshake time $t_{B}=$ $0.45 \mathrm{~ms}$, the density, energy and velocity in $r<R_{B}=200 \mathrm{~cm}$ are modified according to the handshake procedure. The heat loss term uses $T_{S}=8000$ and $t_{S}=4 \mathrm{~ms}$. The most notable difference between the BUCKY simulation and the VTF one is the temperature. Now, we no longer allow the temperature to take the physically unrealistic infinite value at $r=0$, as predicted by the ideal blast model, or the still large values that BUCKY can handle because it posses the appropriate physics to resolve all physical processes at high temperatures. The energy has now been distributed

over all the blast region in a more uniform manner (it is not constant). At $t=2 \mathrm{~ms}$ we observe 
that the shock front is well captured by the hydrodynamic simulation after handshake. There are some notable differences, specially in the inner zone of the chamber (close to $r=0$ ). At $t=4.4$ ms the shock reaches the wall of the chamber and reflects back. This can be seen in the figure, albeit it looks as if the VTF shock reached the wall a bit earlier (a reflection is already there). The maximum temperature at the center of the chamber has dropped to about 9,000 K.

Figure 4 shows the same sequence of event for the second shot. Now, the chamber is no longer in a uniform state (as can be seen in the top figure). This corresponds to $t=62.5 \mathrm{~ms}$. The preheating stage takes place. At $t=62.95 \mathrm{~ms}$ the handshake takes place again. Note that the BUCKY curves plotted in this figure are just for reference since they do not correspond to the flow initialized under the same initial conditions. This figure illustrate that we now posses a handshake model that can be used receptively to simulate multiple shots of the Chamber flows. Overall, the density shows the best agreement between the BUCKY simulations and the VTF thanks to the handshake procedure. 

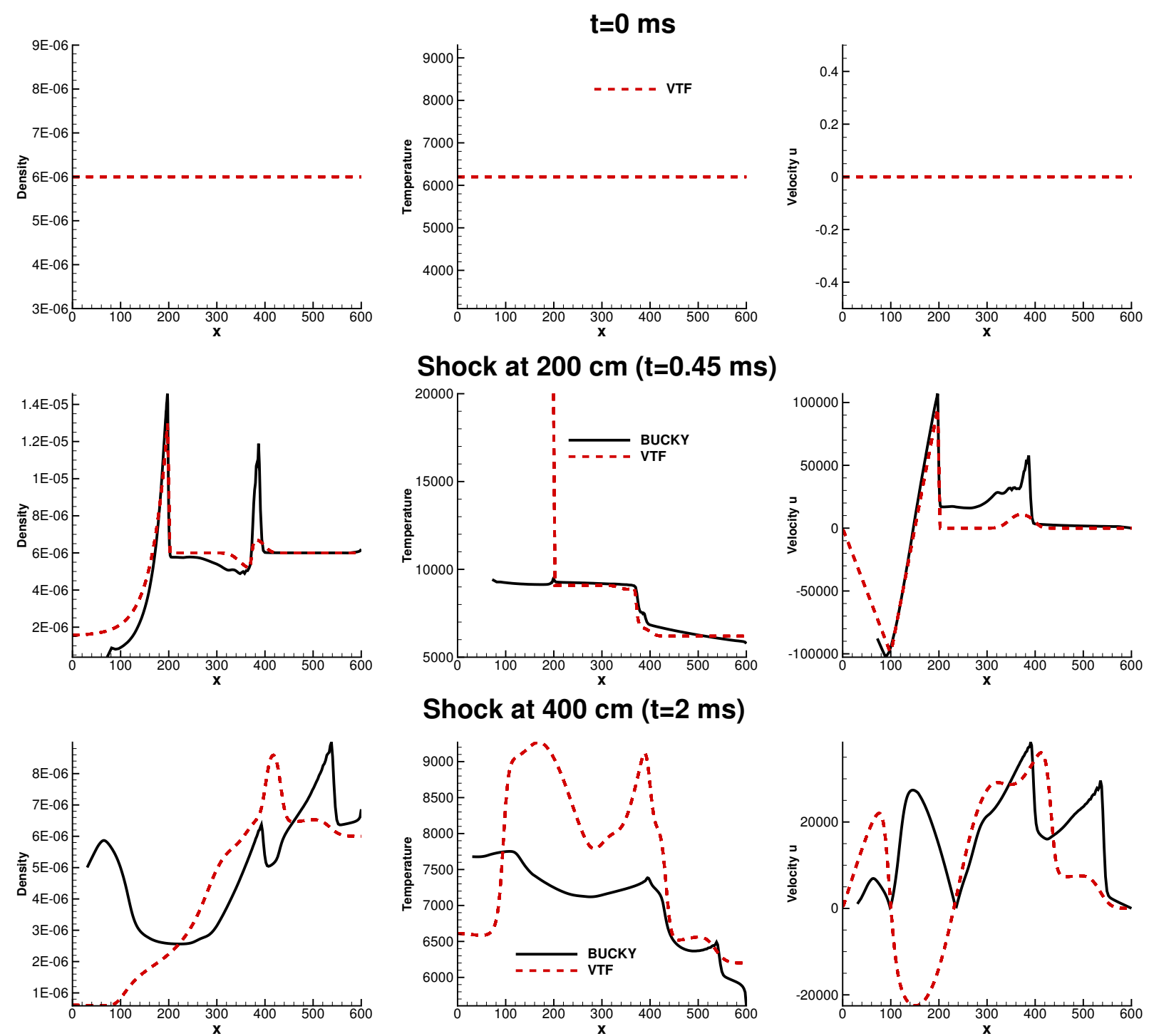

\section{Shock at $400 \mathrm{~cm}$ (t=2 ms)}
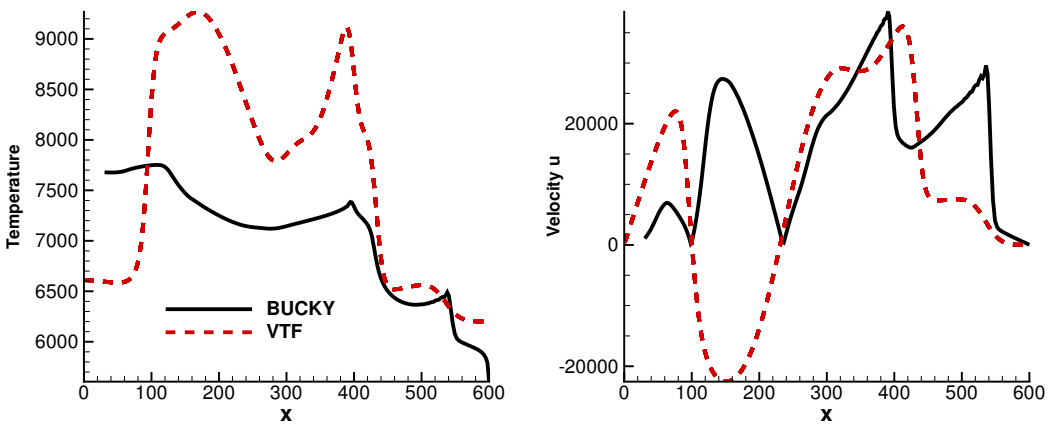

\section{Shock at $600 \mathrm{~cm}$ (t=4.4 ms)}
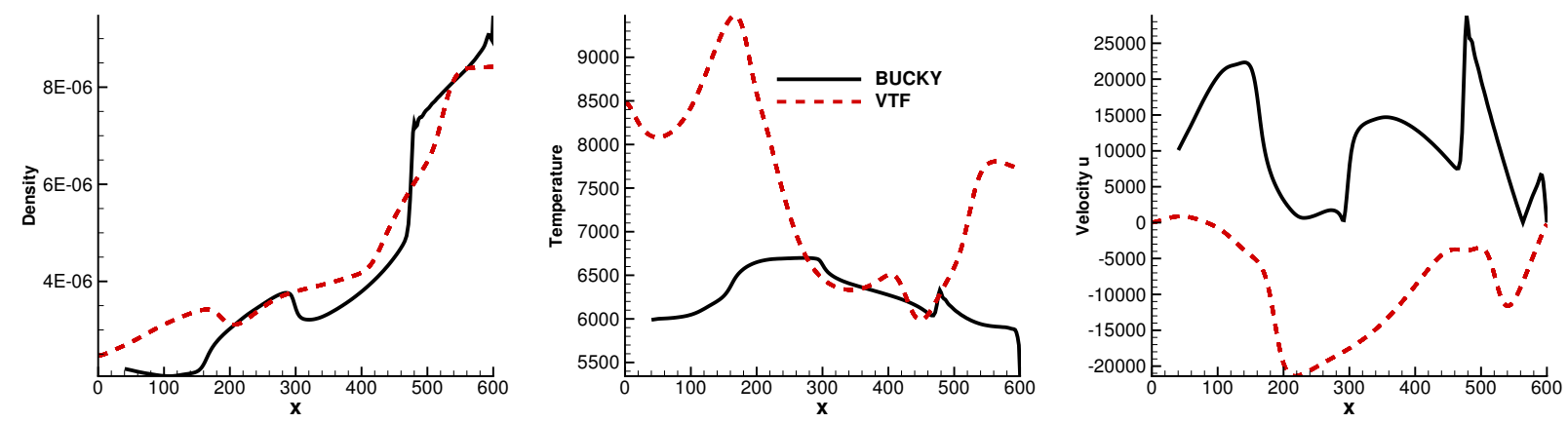

Figure 3: BUCKY \& VTF comparisons different times during the first shot. 

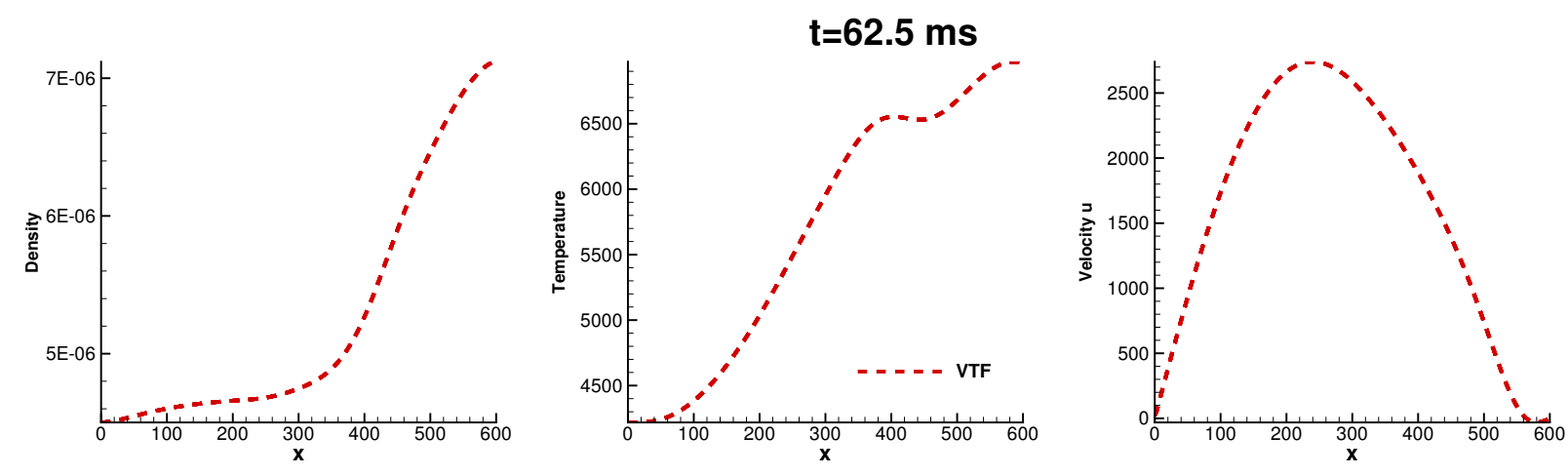

\section{Shock at $200 \mathrm{~cm}(t=62.5+0.45 \mathrm{I}$}
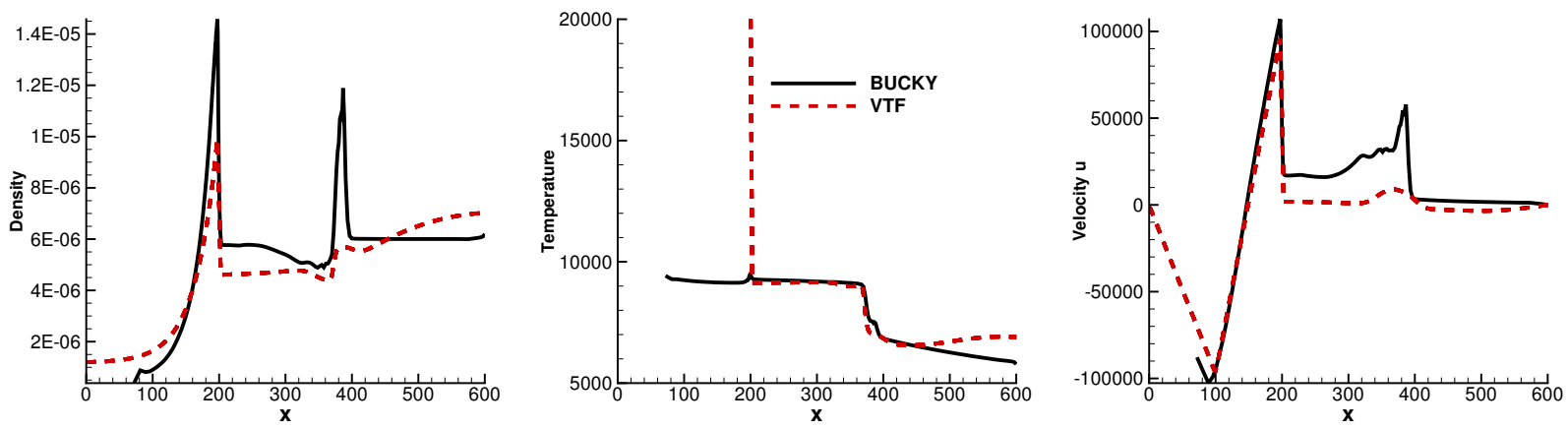

Shock at $400 \mathrm{~cm}(t=62.5+2 \mathrm{~ms}$ )
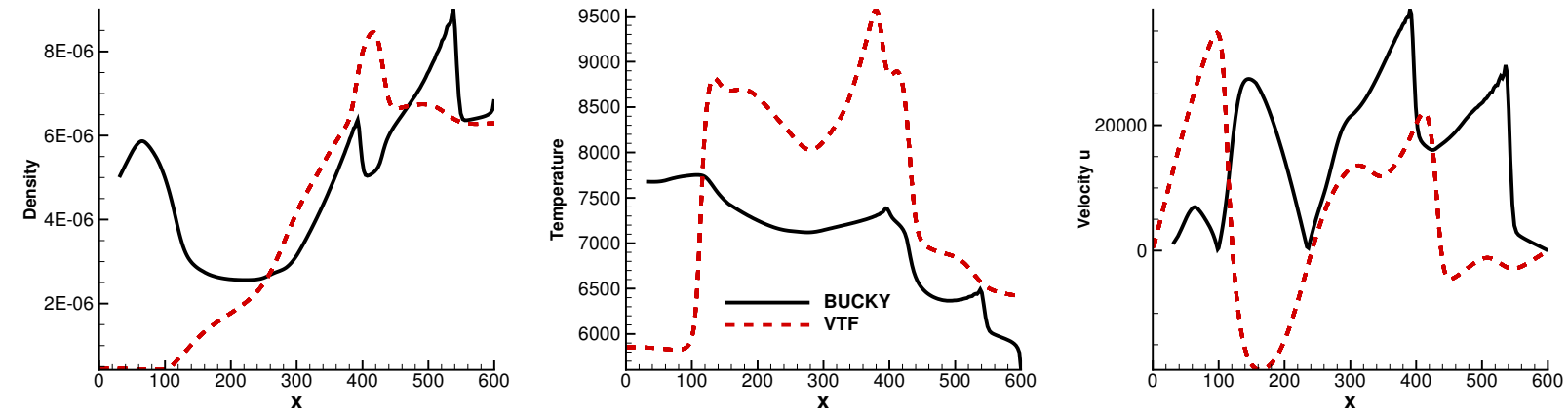

\section{Shock at $600 \mathrm{~cm}(\mathrm{t}=62.5+4.4 \mathrm{~m}$}
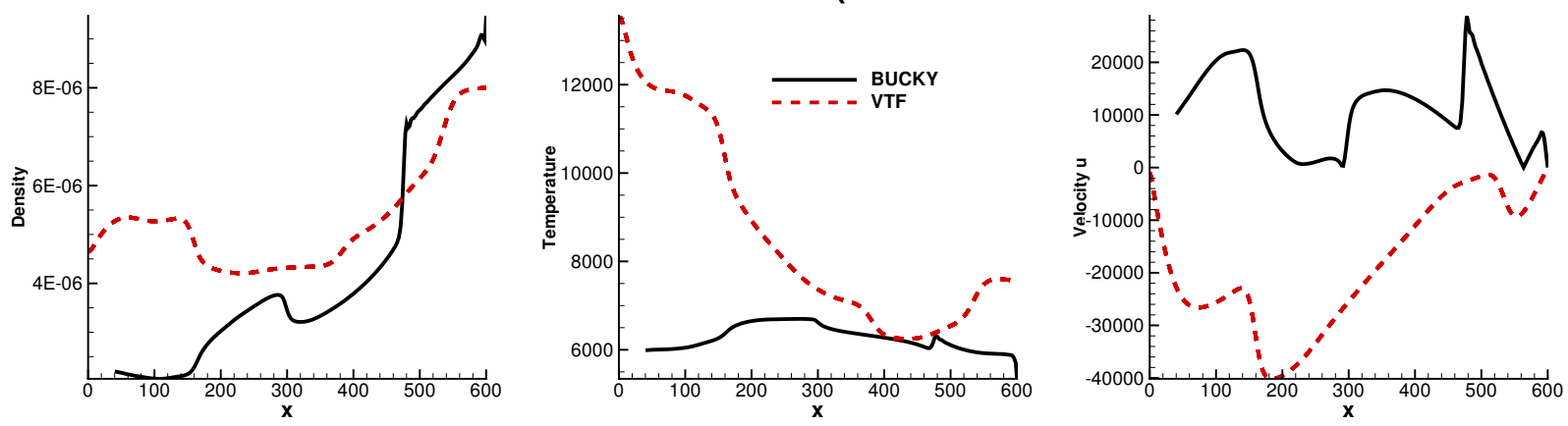

Figure 4: BUCKY \& VTF comparisons different times during the second shot. 


\section{Simulation Description and Parameters}

The common parameters of the chamber simulations are shown in Table 1. The computational domain size is $3200 \times 3200 \times 2200 \mathrm{~cm}^{3}$. The fusion chamber is centered at $(x, y, z)=(0,0,0)$ with the vertical direction aligned with the $z$-axis and the horizontal directions along the $x$ and $y$-axes. The simulation uses 3 levels of grid refinement with the coarsest grid size of $25 \mathrm{~cm}$ and a finest resolution of $6.25 \mathrm{~cm}$. The simulations were performed in Cab and used 256 processors. With this computational arrangement, $7-8$ shots can be completed per week. This report covers three simulations that are geometrically identical but employ different blast intensities as part of a sensitivity study using initial conditions from BUCKY one-dimensional radiation-hydrodynamics calculations. The three simulations are denoted by $0.1 \mathrm{X}, 1 \mathrm{X}$ and $10 \mathrm{X}$, as presented in Table 2 . and indicate the amount by which the radiation multigroup opacities were modified (the simulation labeled $1 \mathrm{X}$ is the nominal case). Figure 5 shows a perspective view of the plane $y=0$ throughout the domain with the chamber (not including the drain pipe at the bottom). Furthermore, we recently started another simulation using one additional level of grid refinement resulting in a fine resolution of $3.25 \mathrm{~cm}$ for the conditions of the $1 \mathrm{X}$ case. This is expected to provide better understanding of small-scale features and more accurate information about debris distribution and the results will be available depending on the availability of the computing resources.

\begin{tabular}{l|ll}
\hline Chamber temperature & 6200 & $\mathrm{~K}$ \\
Chamber density & 6 & $\mu \mathrm{g} / \mathrm{cm}^{3}$ \\
Plenum temperature & 3000 & $\mathrm{~K}$ \\
Plenum density & 12 & $\mu \mathrm{g} / \mathrm{cm}^{3}$ \\
Recirculation flux & 723.82 & $\mathrm{gm} / \mathrm{s}$ \\
Shot period & 62.5 & $\mathrm{~ms}$ \\
Fine Resolution & 6.25 & $\mathrm{~cm}$ \\
\hline
\end{tabular}

Table 1: Common simulation parameters.

\begin{tabular}{l|lll}
\hline & $0.1 \mathrm{X}$ & $1 \mathrm{X}$ & $10 \mathrm{X}$ \\
\hline Preheat radius $(\mathrm{cm})$ & 394 & 370 & 350 \\
Preheat temperature $(\mathrm{K})$ & 10000 & 9200 & 8600 \\
Debris energy (erg) & $9.92 \times 10^{12}$ & $7.84 \times 10^{12}$ & $9.92 \times 10^{12}$ \\
Handshake time(ms) & 0.4 & 0.45 & 0.4 \\
\hline
\end{tabular}

Table 2: Handshake parameters of the different simulations.

\section{Simulation Results}

To better understand the results gathered through this simulation campaign, we have divided the data presentation and analysis into separate sections that focus on particular aspects of the flow. We start by discussing global features of the system (fusion chamber and vacuum vessel), then concentrate on the qualitative features of the flow inside the fusion chamber, followed by a more quantitative analysis of the turbulence and density fields. After these hydrodynamic elements are discussed, we proceed to describe particular aspects of the flow that affect other processes in the 


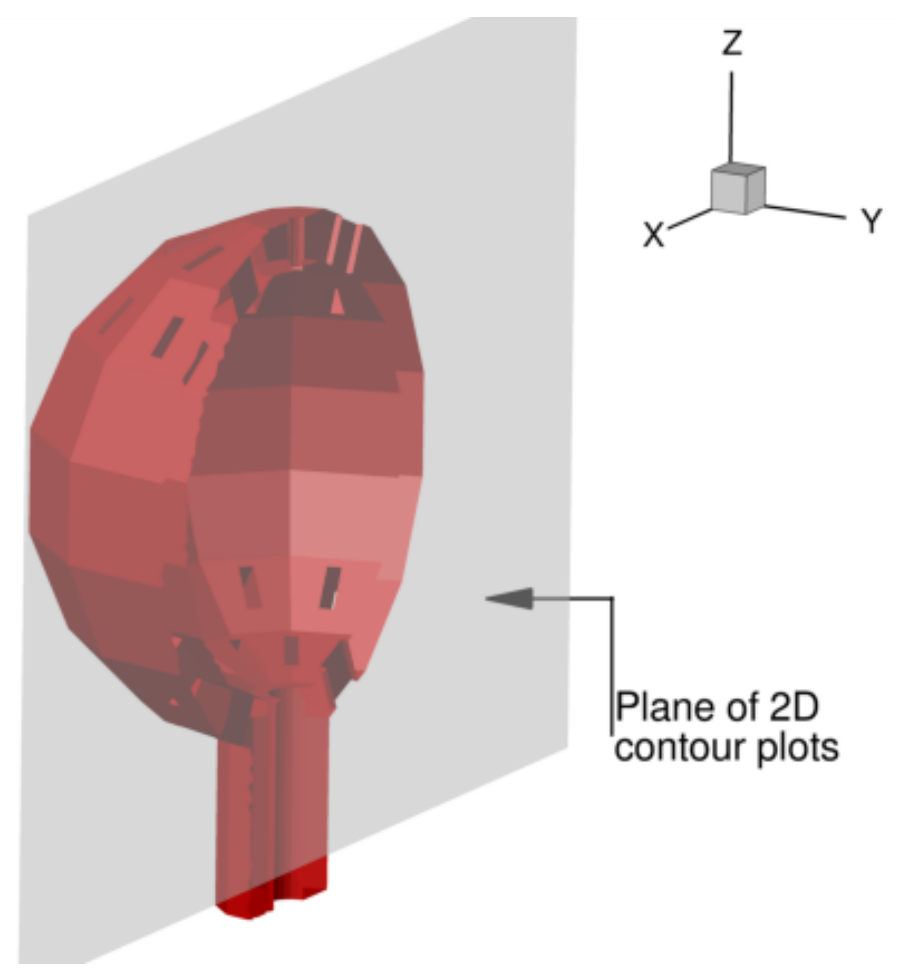

Figure 5: Perspective view of the $y=0$ plane selected to show two-dimensional contour plots of different flow fields.

fusion chamber, including: the environment of the flight path of the target as it is injected into the chamber, the density environment that the laser pathways encounter (according to the simulations), and finally the thermal conditions at the wall of the fusion chamber.

\subsection{Global behavior of the system}

Given the stated objectives of the present simulations, i.e., to achieve a repetitive $\mathrm{n}^{\text {th }}$-state cycle, it was necessary to ensure certain conditions that would allow the flow to exhibit this stable cyclical state. This requires, at least, that mass in the whole system is approximately in balance throughout a cycle. In other terms, that whatever mass enters the system matches the mass that leaves the system. Ideally, we would also desire to achieve energy balance in order to avoid increase or decrease of the mean temperature of the system. While the former requirement is reasonably achievable, the latter (energy) is more difficult with the current simulation technology because it would require accurate heat-transfer balance with the fusion chamber structure, as well as accurate radiation modeling, which is outside the scope of the present effort. In consequence, we focussed on ensuring mass conservation to the best possible degree. One complication in the present simulations is that it is not straightforward (mathematically) to accurately control the inlet/outlet flux of mass through open boundaries because the flow is compressible and there is strong energy deposition taking place in the domain, that generates shock waves that reach these boundaries and can alter locally the direction of flow. Because the inlet/outlets are generally subsonic, and according to the theory of characteristics (valid to a high degree in this high Reynolds number flow), it is not possible to force the mass flux into or out of the domain precisely. The best we can do it to control certain inlet/outlet parameters to drive the flow to desirable conditions. The "controlling" is actually done by employing close loop feedback proportional-integral control theory. In the current 
implementation, there are two controllers operating at all times. The first controller adjusts the pressure of the inlet ports (on the top-bottom surface of the vacuum vessel) so that the total mass flux through the ports is as close as possible to the target clearing ratio described above. Therefore, the inlet pressure $p_{i}$ is obtained by integrating

$$
\frac{d p_{i}}{d t}=-\alpha\left(\iint \rho \boldsymbol{u} \cdot \boldsymbol{n} d S-F_{\text {in }}\right),
$$

where $\alpha$ is the controller gain, $F_{\text {in }}$ is the target recirculation flux over all the inlet surfaces $S$, and $\boldsymbol{n}$ denotes the surface normal incoming into the domain. The second controller is implemented at the drain duct. Its target function is to maintain the total mass in the system (fusion chamber + vacuum vessel) to a given value (in the present simulations, that is the initial total mass). This is achieved by setting the drain velocity through a sponge domain in the whole length of the drain duct by integrating the controller law,

$$
\frac{d U_{d}}{d t}=-\beta\left(\iiint \rho d V-M_{o}\right),
$$

where $\beta$ is the controller gain and $M_{o}$ denotes the initial mass of the system.

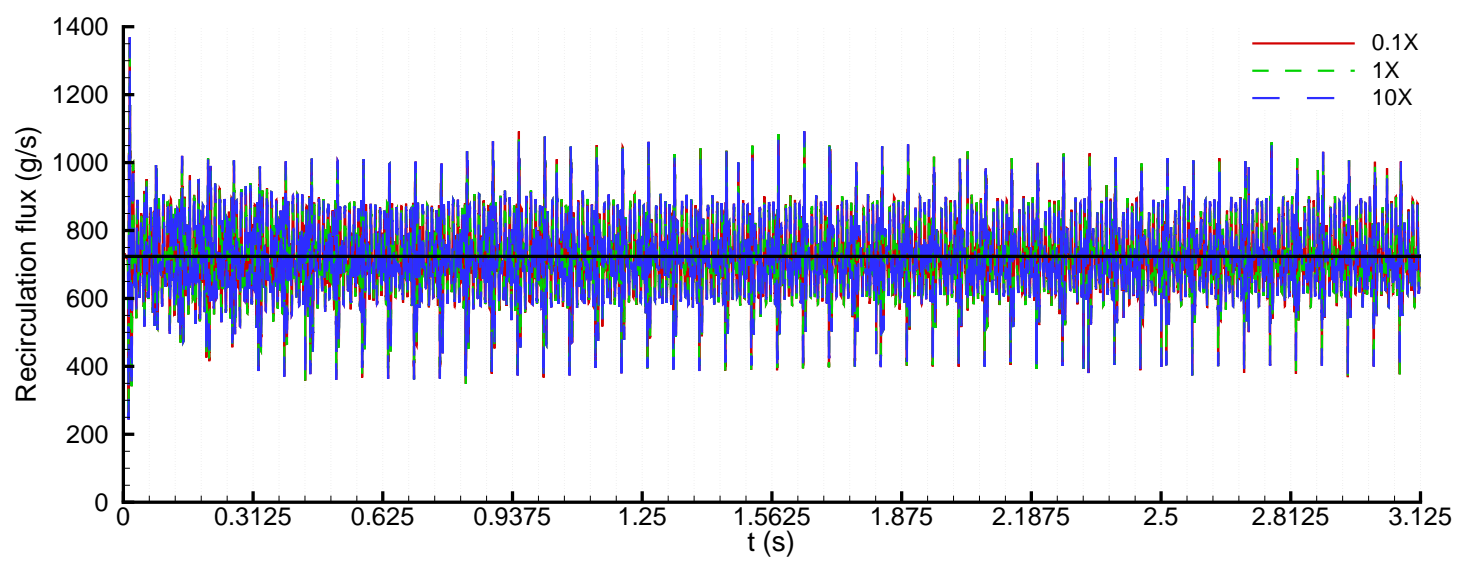

(a)

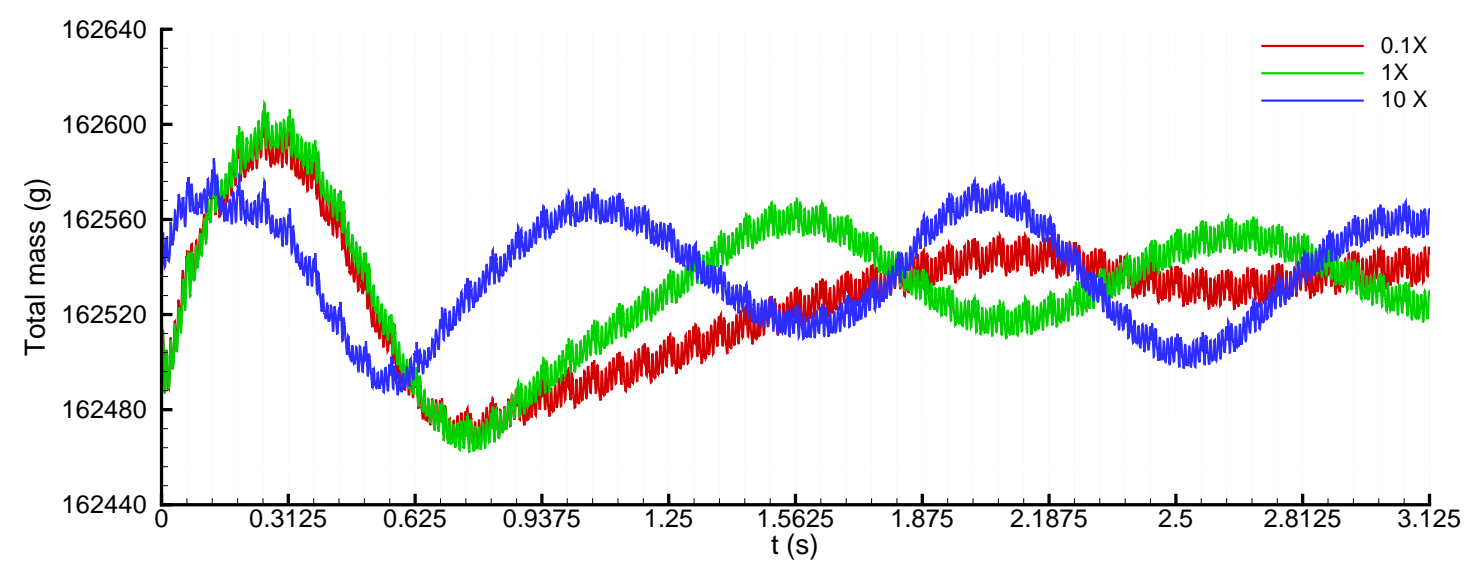

(b)

Figure 6: Conservation of clearing ratio and total system mass by the dynamic controllers.

Figures 6 (a) and (b) show the recirculation flux and total mass, respectively, over 51 shots for all three simulations. In figure 6 (a), the large variation observed in the recirculation flux at 
the beginning of the simulation for all three cases is due to the initial startup of the controller, which washed out quickly. This behavior is a consequence of the first shock wave reaching the walls for the first time through the comparatively quiescent initial flow condition. Later, the flow equilibrates and the recirculation flux becomes more regularized and oscillates around $723.82 \mathrm{~g} / \mathrm{s}$ (which corresponds to approximately $0.83 \%$ clearing ratio with the given shot frequency). The downward and upward spikes overfed in the flux happen when the blast wave introduced at the beginning of each shot reaches the vacuum vessel walls (as a shock wave) and temporarily reverse the direction of flow or when the wave is reflected back it sucks more mass into the vessel, respectively. The controller attempts to balance this dynamic effect by decreasing or increasing the pressure in the inlet. The peak to peak variation of the flux is of the order of $300 \mathrm{~g} / \mathrm{s}$, which in the mean cancel out to produce the mean flux of $723.82 \mathrm{~g} / \mathrm{s}$. Similarly, the drain is operated to adjust the total mass of the domain (vacuum vessel and fusion chamber) to its initial (starting) value. This is presented in figure 6 (b) where very little variation about the initial mass is observed. As the simulations approach the $n^{\text {th }}$ state condition the amplitudes become very small, of the order of \pm 25 $\mathrm{g}$ (over a total system mass of $162 \mathrm{~kg}$ ). We also observe a small amplitude high-frequency variation of the mass of the system which is related to the many temporal scales present in the simulations; the frequency is higher than the shot frequency and it is connected to the reverberating system of shock waves that each shot generates.

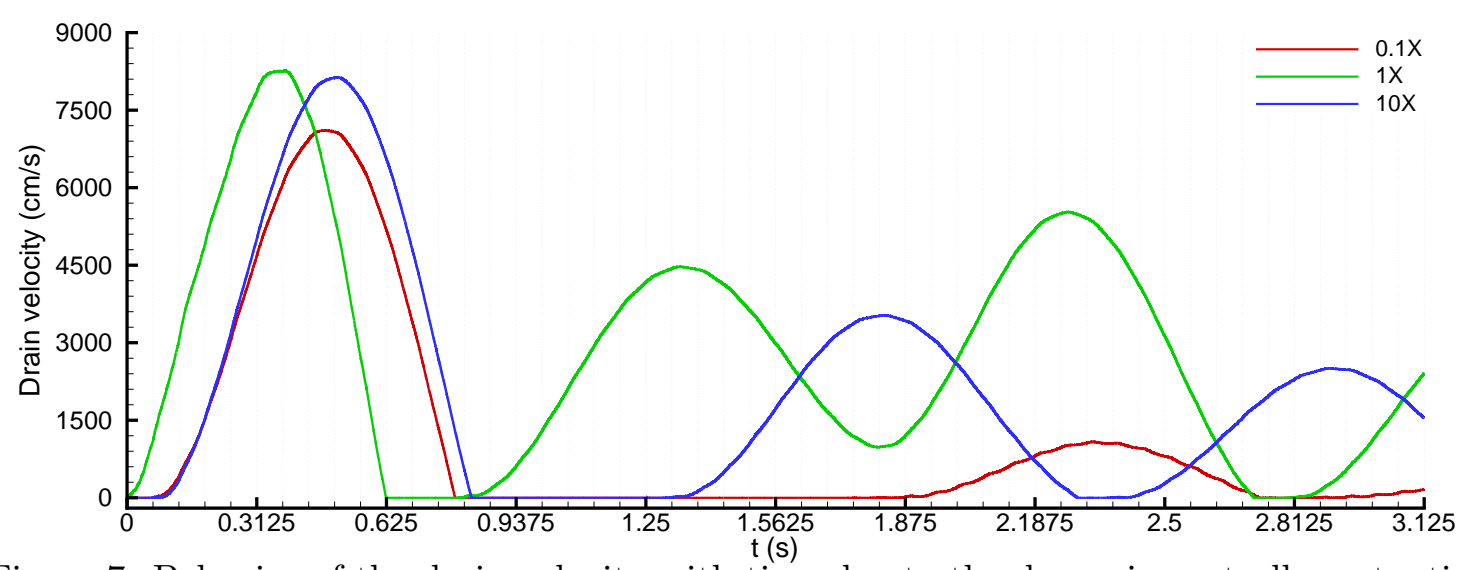

Figure 7: Behavior of the drain velocity with time due to the dynamic controller actuation.

The drain is designed to operate with a finite response time while the total mass exceeds the reference value, see fig. 6(b). Figure 7 shows that the drain opens initially for all three simulations due to initial mass accumulation because the recirculation system is injecting mass from the very beginning. This initial drain of mass is too strong in all simulations and eventually leads to the shutting down of the drain once the total mass reaches the target mass. Since the flow is compressible, even if the controller attempts to close the drain there is still some mass able to leave the domain (because of the flow inertia in the drain pipe and optical ports on the vacuum vessel) and this allows some mass to scale the system for some time. Eventually, the conditions reverse and the drain is able to open up again and evacuate fusion chamber fluid out of the system. The response of the system is too slow for the drain controller to achieve a permanently open state even after more than 50 shots, but there is evidence in the figure that it will eventually; the $0.1 \mathrm{X}$ simulation has the highest effective drain response time, despite the controller constants being the same.

As a consequence of the flux injected into the system through the ports of the vacuum vessel and the drain controller that prevents accumulation of mass in the system, the fusion chamber 


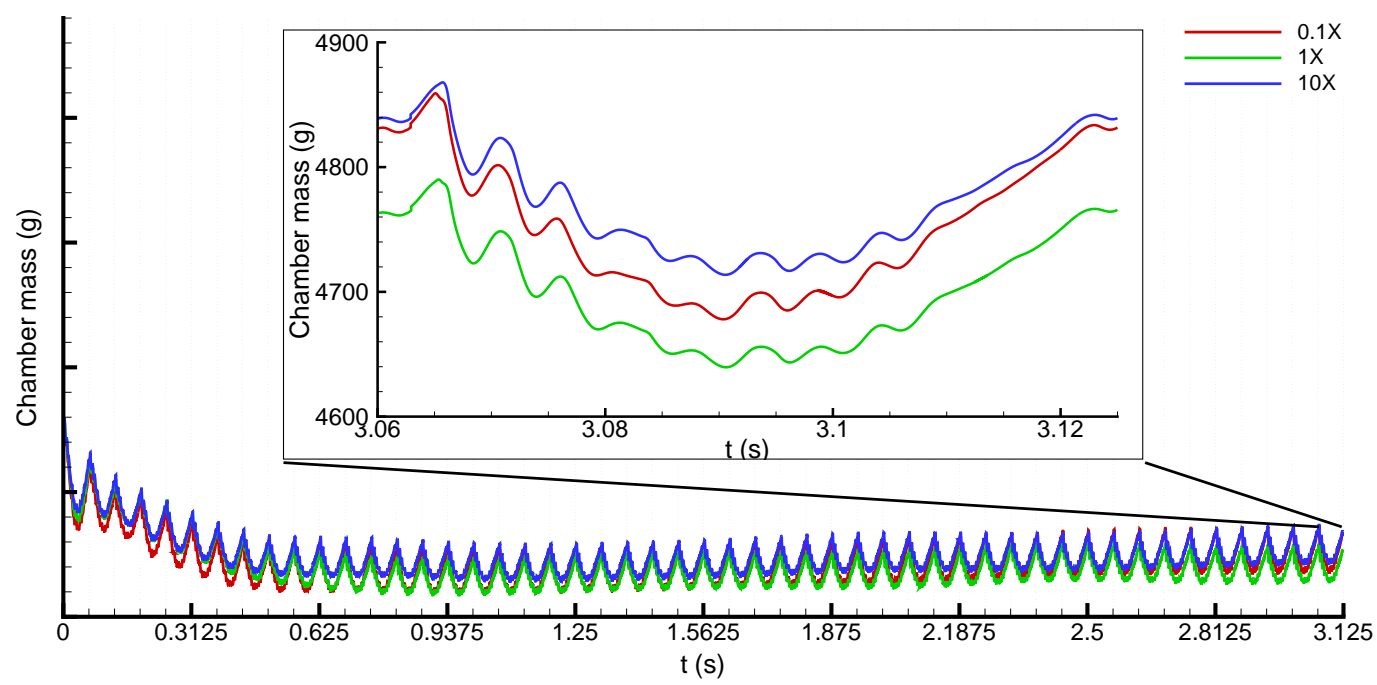

Figure 8: Evolution of chamber mass over 51 shot cycles with inset denoting the details of the variation over one shot towards the end of the simulations.

interior develops a slight pressure deficit that ensures that on the mean there is a net influx of fresh gases from the vacuum vessel into the chamber. Of course, this mean pressure deficit is temporarily disturbed during the period that follows the shot, but the perturbation dies down and the system goes back to the starting point. The variation in chamber mass as a function of time is shown in figure 8. After the initial blast, $7-8 \%$ loss in fusion chamber mass is observed over approximately the first 10 to 12 shots. This is the mass that the initial blasts eject outside the fusion chamber (and that also help lowering the pressure in the chamber). Then the chamber mass increases a little and gradually reaches a more or less stable state (there is a small net increase of mass over time that might be related to the fact that there is no perfect energetic balance in the system, which we expect to be observable in long time scales). If we concentrate on one of the later cycles, the inset in figure 8, the fusion chamber loses some mass after each shot, of the order of $250 \mathrm{~g}$, and this continues for about half of the shot period. Then, the conditions are reversed due to the overpressure in the vacuum vessel resulting in a net fusion chamber mass increase, that takes the chamber back to the starting conditions just before the shot. This process is repeated every cycle. The inset also shows that the first two thirds of the cycle is more active than the last third of the period, likely due to the many reflections that the initial bast wave experiences inside the chamber. This observation also suggests clearly that for the given energy levels we used, reducing the shot period (increasing the frequency) by more than $1 / 3$ might produce flow conditions such that the shock-wave reverberation generated by one shot might no have sufficient time to die down before a new blast wave is produced. This most likely will result in quite different turbulence levels in the chamber since a period of turbulence decay may not take place before a new shot. This energetic turbulence state was not explored in the simulations since only on firing frequency was investigated.

Figures 9 (a) and (b) show the mass and debris flux, respectively, out of the fusion chamber through the combined optical ports and the drain pipe over 51 shots (positive values are fluxes out of the chamber). Over the first 10-12 shots fluid is pushed out of the chamber which is consistent with figure 8. All simulations show a similar behavior in the mass flux, with substantial variation during each cycle; peak instantaneous flux of $60 \mathrm{~kg} / \mathrm{s}$ with an obvious skewness towards positive fluxes (in the mean, the average is approximately zero). Very small quantity of debris is observed to 


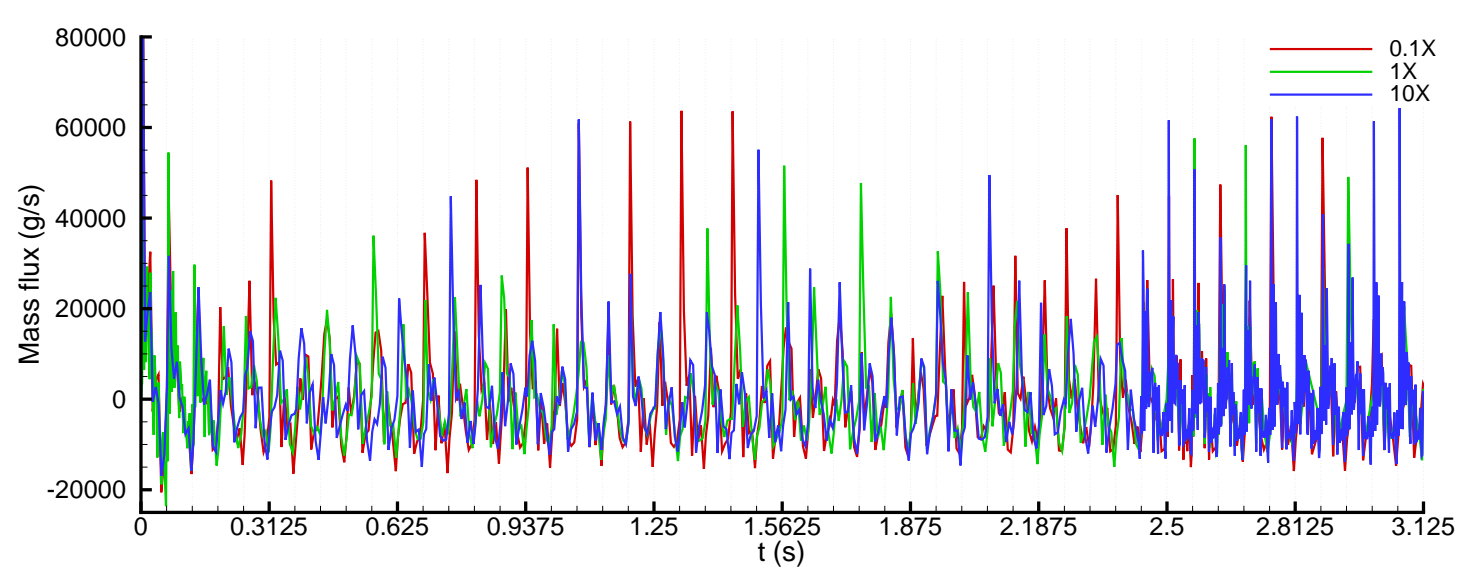

(a)

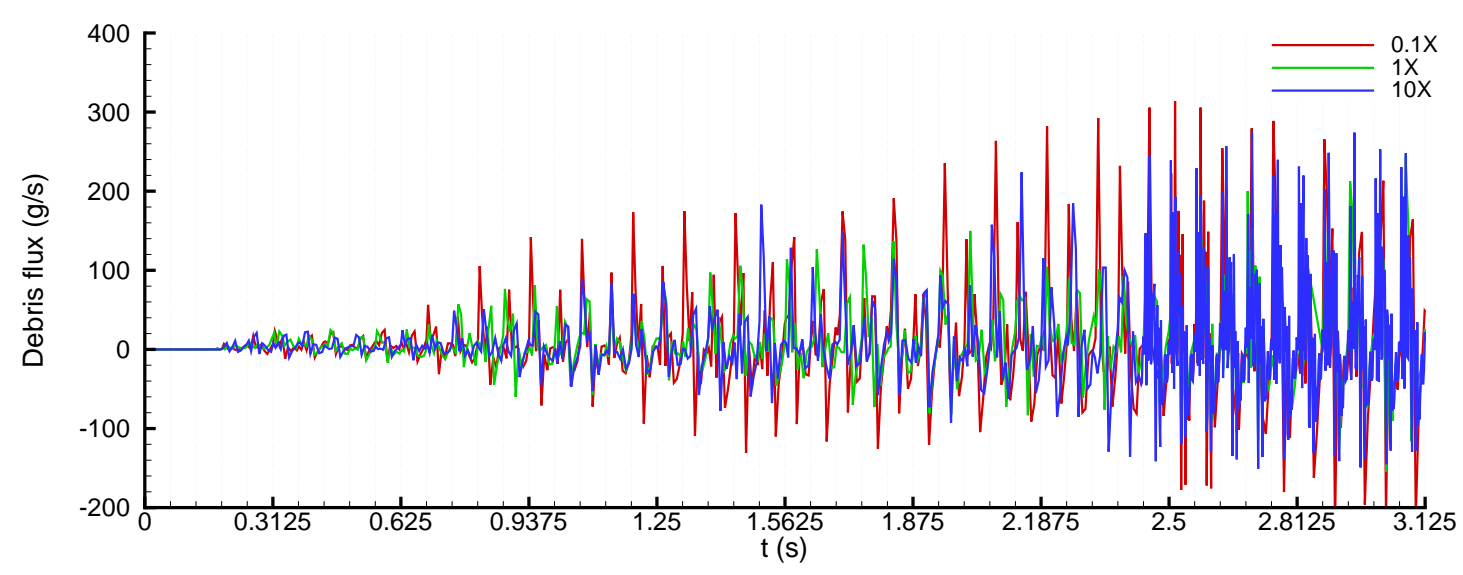

(b)

Figure 9: Total fusion chamber mass and debris fluxes over 51 shot cycles.

move in or out of the chamber over the first few shots. Later, as more shots are accumulated, more debris flux occurs toward the vacuum vessel. Higher peaks of debris flux is observed for the 0.1X simulation, consistent with the more chaotic flow distribution presented in figure 12 (a) (below).

Figure 10 shows the total mass and debris that leaves the ports (excluding the drain) as a function of time. Although, a significant fraction of the fluid mass is being pushed in and out of the fusion chamber instantaneously due to the reverberating blast waves, the integrated amount of mass flow through the ports while starting positive (the initial emptying of the fusion chamber due to the fist few blast waves) they eventually become negative, i.e., there is a net incoming of fresh vacuum vessel fluid, which is the objective of the recirculation system. Simulation 0.1X takes longer to achieve a comparable state as that of the other simulations. Figure 10(b) shows that over 51 shots, 4 to $6 \mathrm{~g}$ of debris has left the fusion chamber throughout the ports (not the drain, as intended). This is less than 2 target masses (each of $3 \mathrm{~g}$ ) over 51 injected over all the time, or about $4 \%$ debris loss. 


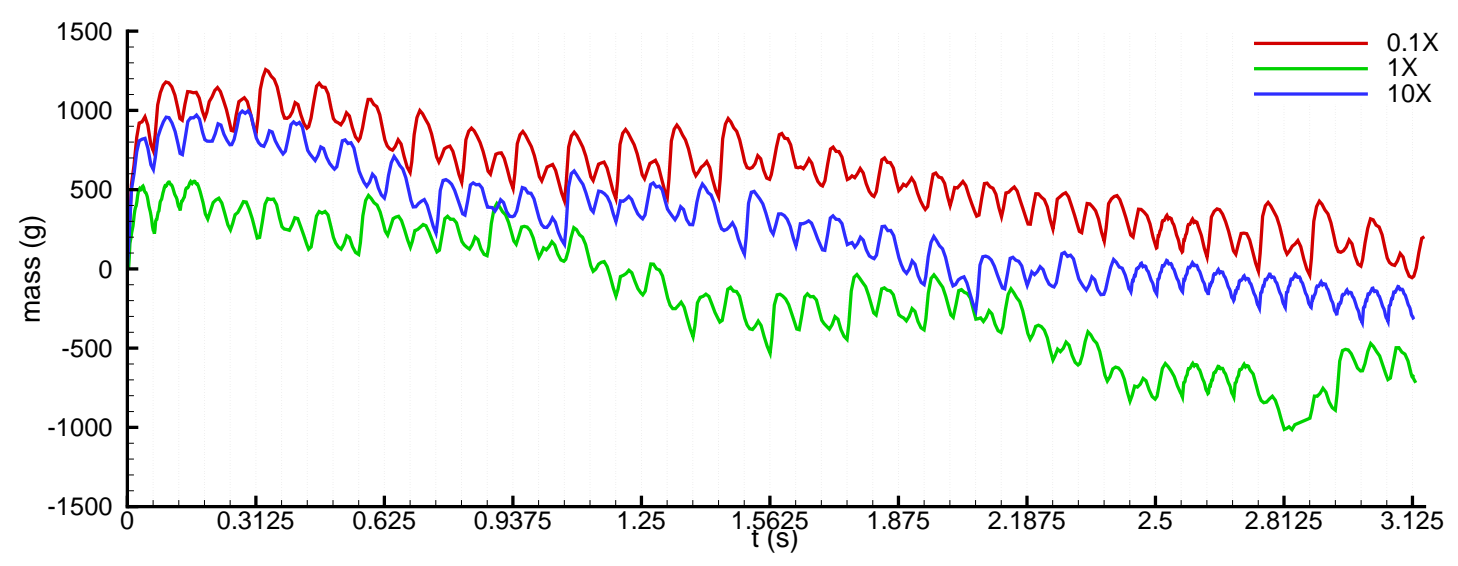

(a)

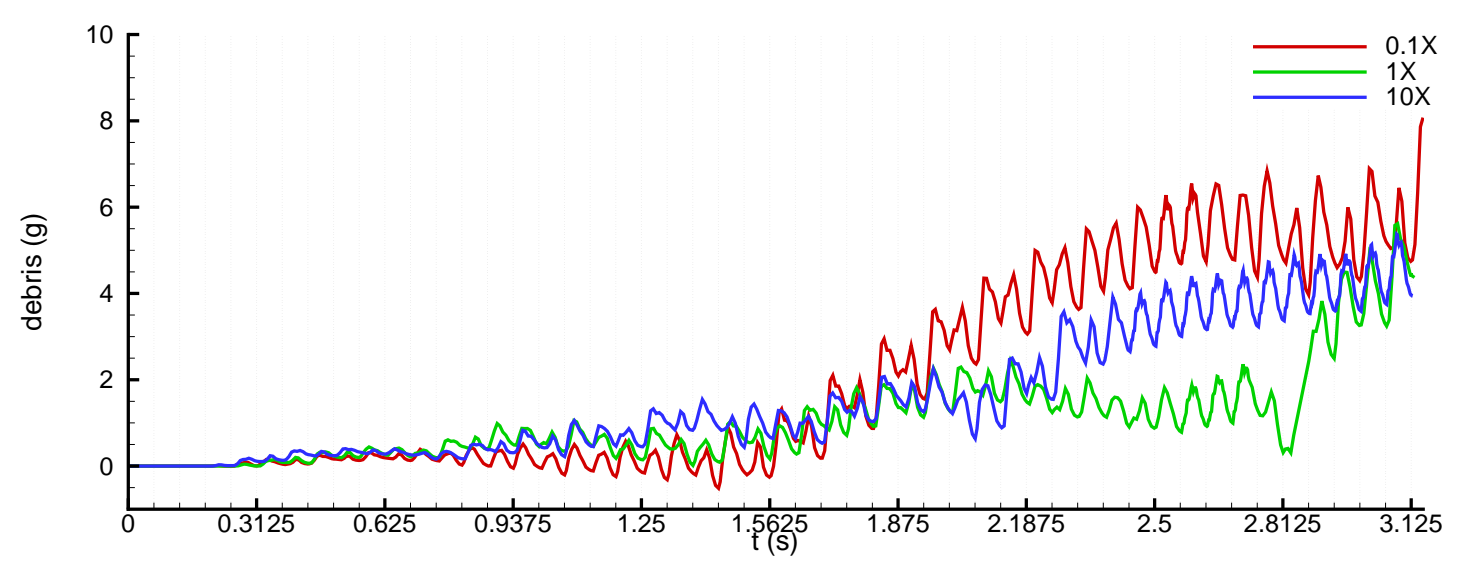

(b)

Figure 10: Integrated chamber mass and debris fluxes over 51 shot cycles only through 49 ports (excluding drain). 


\subsection{Approaching the $\mathrm{n}^{\text {th }}$ shot state}

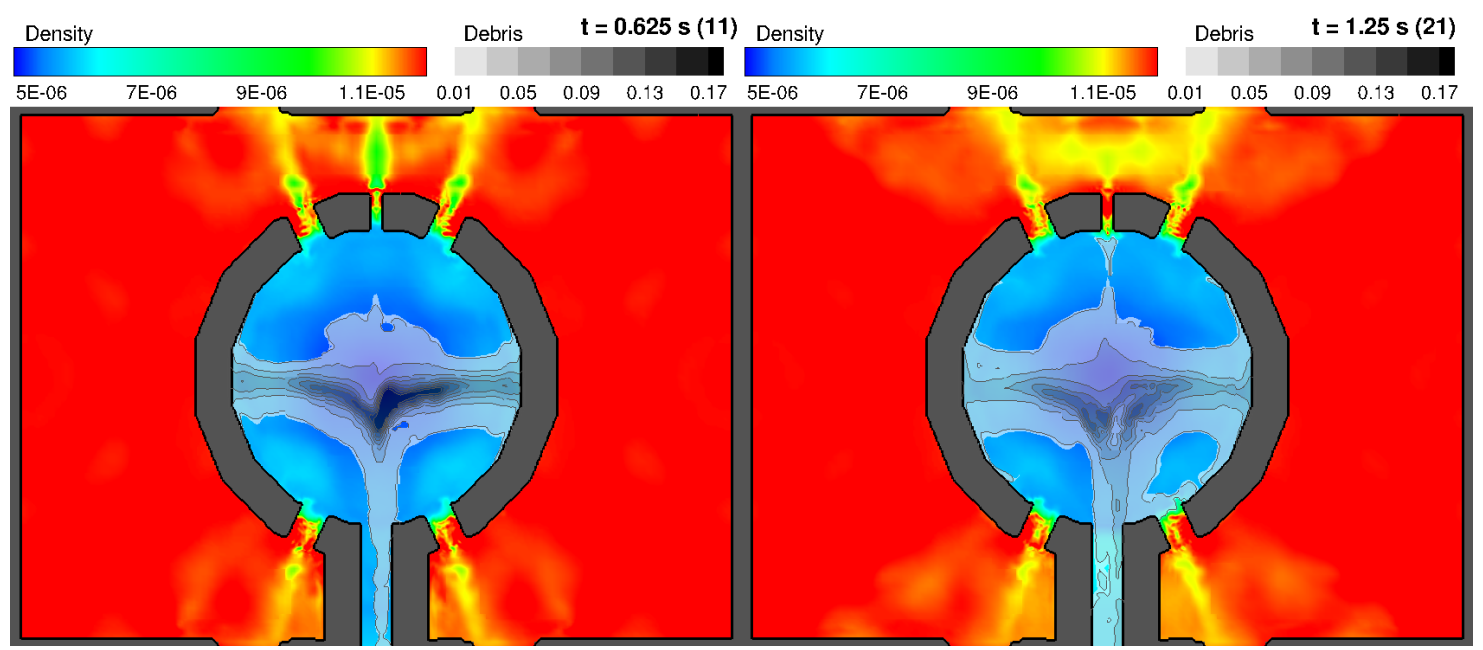

(a) $11^{\text {th }}$ shot

(b) $21^{s t}$ shot

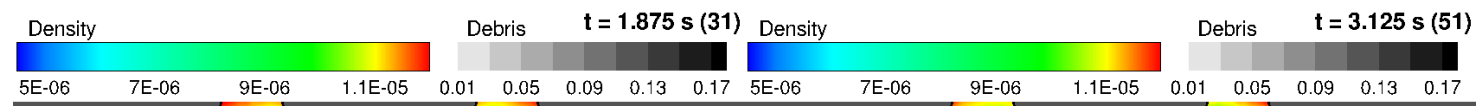

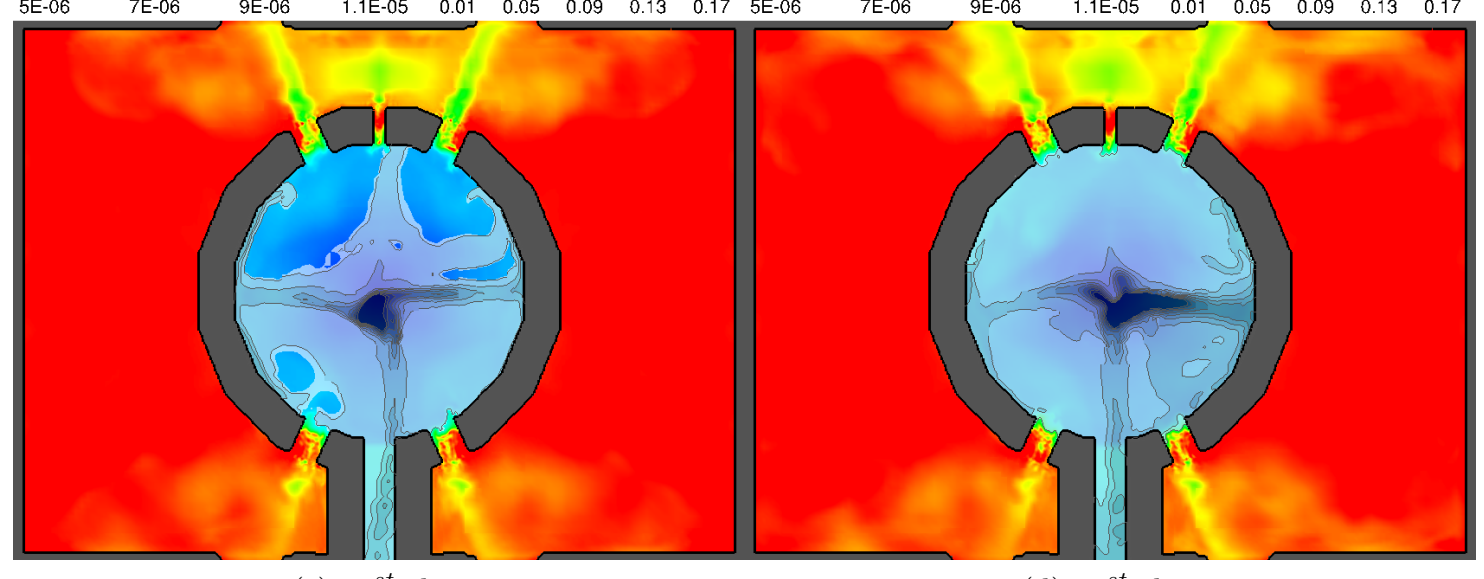

(c) $31^{\text {st }}$ shot

(d) $51^{\text {st }}$ shot

Figure 11: Contours of density $\left(\mathrm{g} / \mathrm{cm}^{3}\right)$ and debris mass fraction on the $y=0$ plane shortly before $11^{\text {th }}$ (a), $21^{\text {st }}$ (b), $31^{\text {st }}$ (c) and $51^{\text {st }}$ (d) shot, respectively, for the $1 \mathrm{X}$ simulation. Density is shown in color isocontours and debris is shown in gray isocontours and black isolines.

The main focus of this report is the investigation of the state of the flow inside the fusion chamber in the cyclical state that is achieved after a sufficiently large number of shots has taken place, what we call the $\mathrm{n}^{\text {th }}$-shot state. Figure 11 shows contour plots of density and debris mass fraction shortly before the $11^{\text {th }}, 21^{\text {st }}, 31^{\text {st }}$ and $51^{\text {st }}$ shots for the $1 \mathrm{X}$ simulation. Density is shown using colored contours from blue to red, denoting low to high density, respectively (equivalently, high to low temperature). Debris is shown based on mass fraction, i.e., partial density of debris divided by total density. In general, the following sequence of events takes place during and after each energy deposition in the simulation, that is referred here as a shot. Initially, a blast wave emanating from the center of the fusion chamber pushes the gas radially outward. Being reflected from the chamber walls, the blast wave creates a 'reverberating' pattern of waves that alternately push 'hot' gas out and pull 'cold' gas in through the optical port openings. Shortly before the $11^{\text {th }}$ 
shot, the amount of hot and low density gas contributing to the mixing of chamber and vacuum vessel fluids through the ports by the 'push-pull' process covers a relatively small region near the top and bottom of the vacuum vessel. By the time we reached 51 shots, a larger amount of mixed gas in the vacuum vessel is observed. Some of the fluid even reaches to the vacuum vessel ports (location of the optical ports in our simulation) where the incoming recirculation flow is specified based on the clearing ratio. Due to the presence of the drain, less chamber-vacuum vessel fluid is mixed near the bottom of the vacuum vessel. The effect of the drain is clearly visible in the debris contours. The debris concentration is alternately being compressed and expanded by the blast wave and being sucked through the drain; which is the intention of the drainage system. Most of the debris is found to be concentrated in the equatorial region. Although, some fraction of the debris is observed everywhere inside the fusion chamber by 51 shots; an insignificant amount of debris is observed to be expelled to the vacuum vessel (this is based on the current simulation results up to 51 shots, nothing is inferred about mixing at much longer timescales $\sim$ thousands of shots).

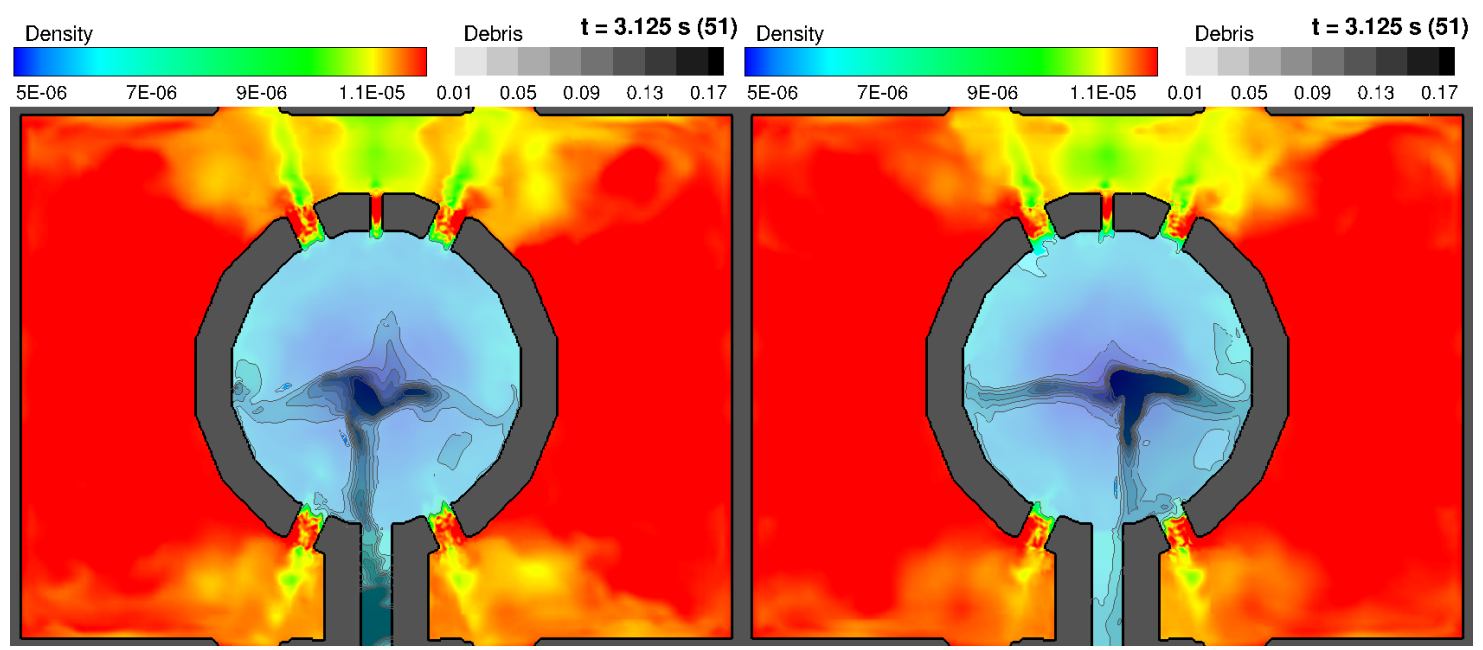

(a) $0.1 \mathrm{X}$

(b) 10X

Figure 12: Contours of density $\left(\mathrm{g} / \mathrm{cm}^{3}\right)$ and debris mass fraction on the $x-z$ plane shortly before $51^{\text {st }}$ shot for $0.1 \mathrm{X}$ (a) and 10X (b) simulations, respectively. Density is shown in color isocontours and debris is shown in gray isocontours and black isolines.

Figures 12 (a) and (b) show the density and debris contours for the $0.1 \mathrm{X}$ and 10X simulations, respectively, before the $51^{\text {st }}$ shot. Due to the larger preheat radius and preheat temperature of the $0.1 \mathrm{X}$ simulation, see table 2, we observe stronger mixing of chamber and vacuum vessel fluids. This is consistent with the more chaotic (distorted) distribution of debris that is observed inside fusion chamber in comparison with the other two cases. In this figure, both for the $0.1 \mathrm{X}$ and $10 \mathrm{X}$ cases and to a lesser extent in the $1 \mathrm{X}$ simulation discussed in the preceding paragraph, we also observe that the column of debris that is supposed to exit the domain through the drain duct in the bottom half of the chamber center can meander and impinge on the walls of the fusion chamber in between the nearest optical port opening and the drain duct. This phenomenon increases the opportunities for debris to exit through the optical port opening into the vacuum vessel, although the lower pressure in the fusion chamber minimizes the instances when the debris can reach the vacuum vessel. The fluid mechanical reasons for this observation is rather simple. Once the column of debris starts to oscillate laterally (due to the strength of the blast wave interactions and nonuniformities of the flow leaving the domain through the drain duct) and impacts on the walls of the chamber, a stagnation or impinging flow configuration will split the column of debris sending 
part of the flow along the wall chamber (climbing) along the wall until an opening is encountered. Now, this impinging phenomena does not happen always at the same location on the chamber wall since the debris column will tend to spin around the $z$ axis in a more or less spiraling manner (assuming the usual jet-like instability patterns are taking place here). This means that it was rather fortunate that the behavior is observed clearly in figures 12 (a) and (b) at the $51^{\text {st }}$ shot for simulations $0.1 \mathrm{X}$ and 10X, and it also explains what it is only seen clearly in figure 11(c) at the $31^{\text {st }}$ shot for simulation $1 \mathrm{X}$ and not in the other subfigures at other shot times. An important question is what produces this meandering of the main column of debris. This can be answered by looking into the behavior of the drain controller in figure 7. Since the objective function of the drain controller is to maintain the mass in the whole system to a balanced state, it does occur that from time to time the drain is shut off. Under such conditions, the debris column has no choice but to seek other routes to carry its momentum, since the drain is not a viable outlet during those periods; see comments about the drain controller in $\S 4.1$. Therefore, it seems that it would be more desirable to employ a specialized, or more intelligent, controller that is capable to regulating the mass in the fusion chamber without ever shutting off completely. Otherwise, we risk generating the conditions observed in figures 12 by the temporary closure of the drain.

\subsection{Hohlraum Environment}

To understand the flow environment around the hohlraum (or target) flight path, we have plotted different quantities along the trajectory from the center to the top opening of the fusion chamber for all three simulations in figure 13 . All three simulations show approximately the same behavior. The temperature profile (a) shows a rise (when approaching from the top) at approximately the same location of $r=600 \mathrm{~cm}$. This is consistent with the corresponding drop in density (b). Figure 13(c) and (d) show the axial (along the hohlraum nominal flight direction) and lateral velocities along the same path. Peak axial velocity is of the order of $\pm 4000 \mathrm{~cm} / \mathrm{s}$, except for the $0.1 \mathrm{X}$ simulation which shows a large dip of about $8000 \mathrm{~cm} / \mathrm{s}$. Here, negative velocity implies a velocity in the direction of flight of the hohlraum and positive velocities implies a velocity against the flight direction. The lateral velocities, those most likely to affect the lateral displacement of the hohlraum, can be as large as $2000 \mathrm{~cm} / \mathrm{s}$. Based on preliminary design conditions of injection velocities of the hohlraum of $250 \mathrm{~m} / \mathrm{s}(25,000 \mathrm{~cm} / \mathrm{s})$, the observed lateral velocities are very small (less than $10 \%$ of flight velocity). One element that might be considered is that the density in the initial segment of the flight path (within the vacuum vessel) is high, of the order of $12 \mu \mathrm{g} / \mathrm{cm}^{3}$ (in comparison with target flight design estimates of $6 \mu \mathrm{g} / \mathrm{cm}^{3}$ ), which will result in higher local Reynolds numbers for the hohlraum and might allow the possibility of vortex shedding from the back of the target and, possibly, substantial oscillatory lateral forces. These could be compensated by lowering the injection velocity or by spinning the target to stabilize the wake. 


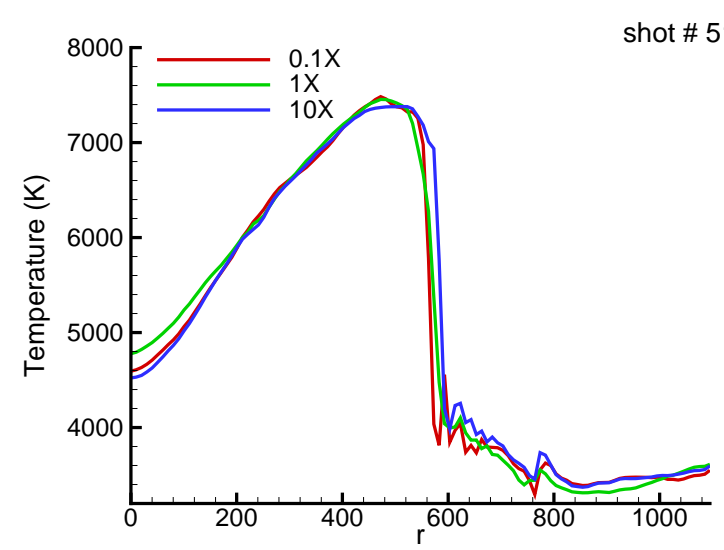

(a)

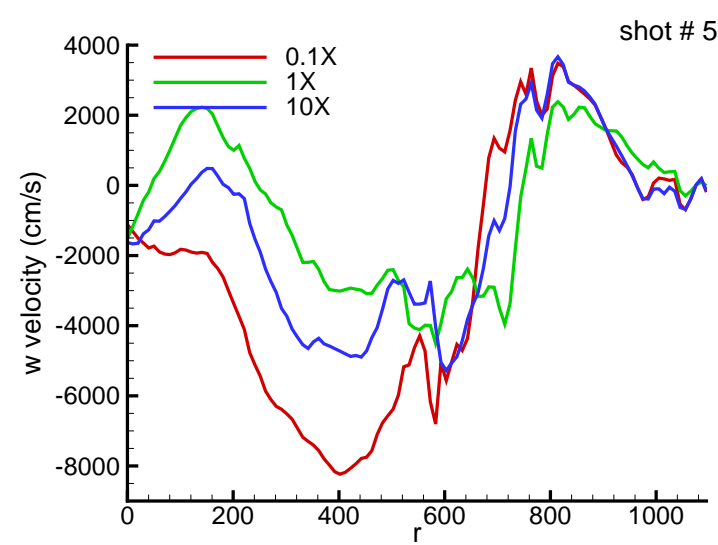

(c)

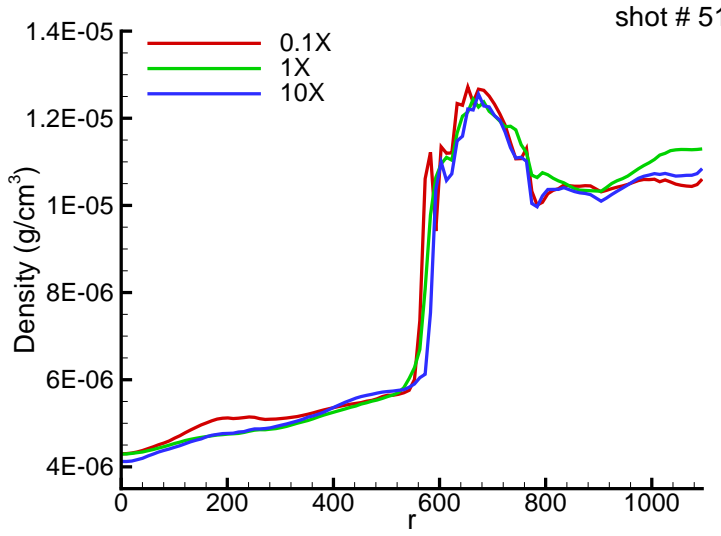

(b)

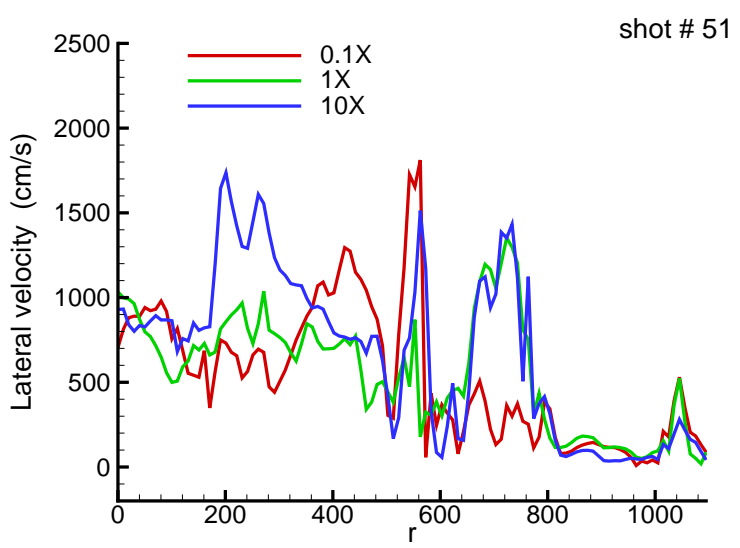

(d)

Figure 13: Comparison of temperature (a), density (b), w velocity (c) and lateral velocity (d) along the line of target flight shortly before the $51^{s t}$ shot. Distance, $r=0$ denotes the center of the fusion chamber. 


\subsection{Turbulence Spectra}

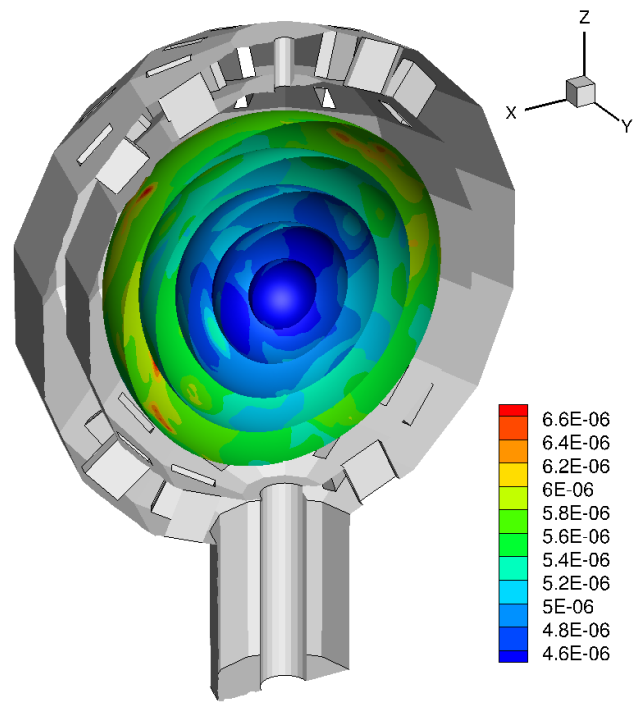

(a) $0.1 \mathrm{X}$

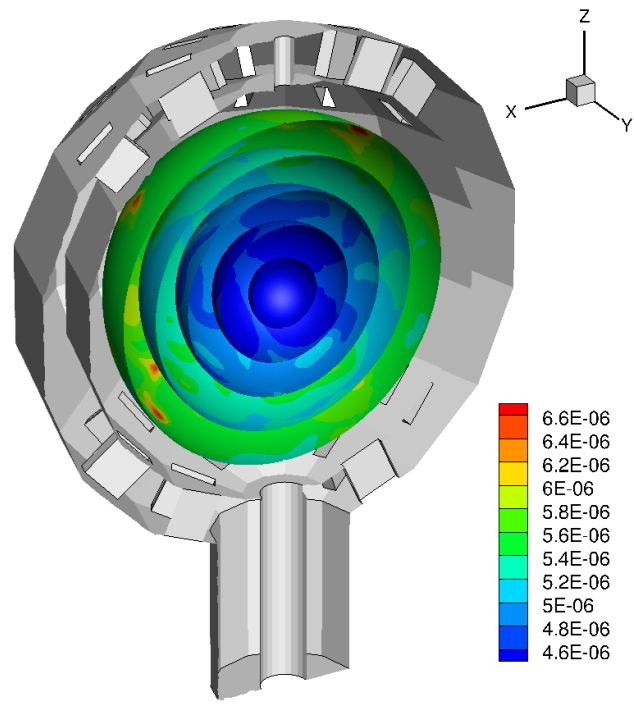

(b) $1 \mathrm{X}$

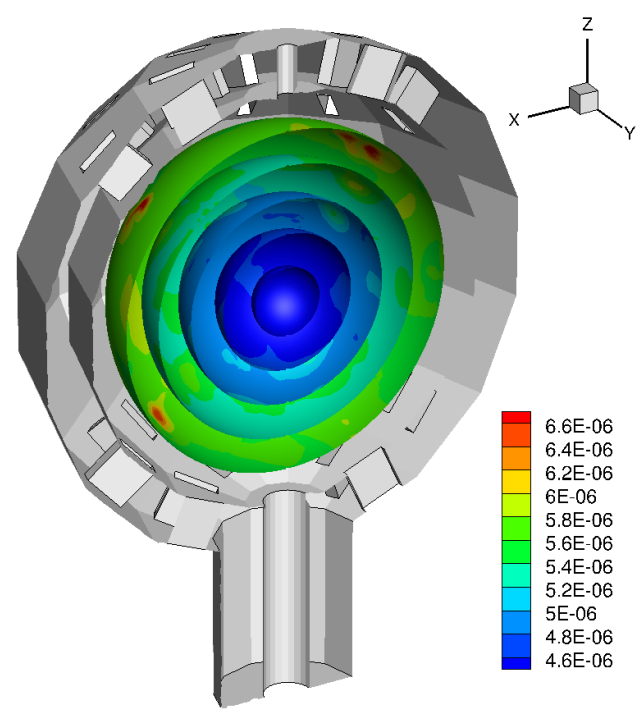

(c) $10 \mathrm{X}$

Figure 14: Density contours interpolated on spherical shells inside the fusion chamber shortly before the $51^{\text {st }}$ shot for the $0.1 \mathrm{X}(\mathrm{a}), 1 \mathrm{X}(\mathrm{b})$ and $10 \mathrm{X}$ (c) simulations, respectively. Units of density are $\mathrm{g} / \mathrm{cm}^{3}$.

The density and velocity was collected on eleven spherical shells at varying radial distance from 50 to $550 \mathrm{~cm}$ inside the fusion chamber. Figure 14 shows the density in these shells for the three simulations just prior to the $51^{s t}$ shot. Figure 15 shows the averaged density, density intensity and kinetic energy on those spherical surfaces shortly before the $51^{\text {st }}$ shot. Density profiles are similar in the three simulations and the only difference in behavior is on the turbulence kinetic energy for simulation $0.1 \mathrm{X}$, which is higher, and consistent with our previous observations of stronger turbulence in the fusion chamber for this case. Peak turbulence intensity for the $1 \mathrm{X}$ and $10 \mathrm{X}$ simulations is of the order of $4000 \mathrm{~cm} / \mathrm{s}$ and $5500 \mathrm{~cm} / \mathrm{s}$ for the $0.1 \mathrm{X}$ simulation. Density root- 
mean-squared (r.m.s.) is of the order of $\% 5$ of the mean. Comparing figure 15 (a) with figure 13 (b) show that the mean density is very close to the instantaneous density over the openings of the fusion chamber (at $r \sim 600 \mathrm{~cm}$ ). These regions can be seen in figure 14 where the regions just inside the fusion chamber near the ports have higher density (yellows and reds) as opposed to the regions underneath the openings which have relatively smaller densities (blues); although the variation in density is small as shown in the r.ms. figure. The high density comes from the fluid of the vacuum vessel through the mass exchanges that are induced by the blasts and recirculation system.

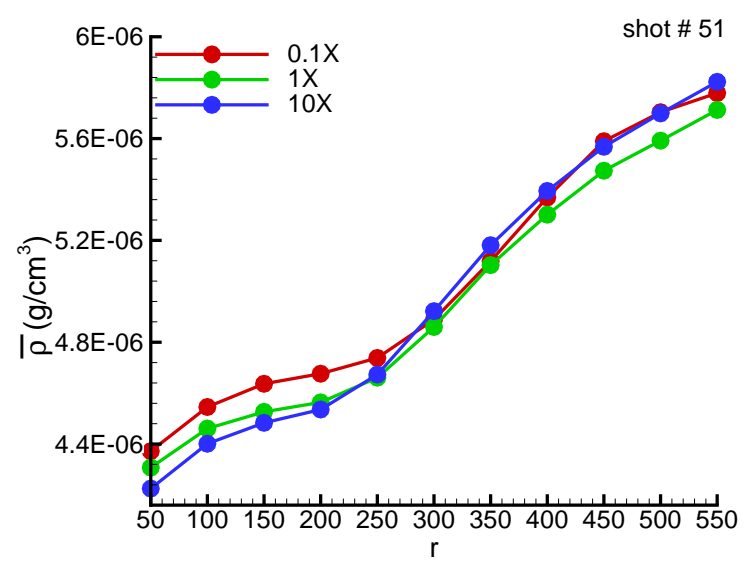

(a)

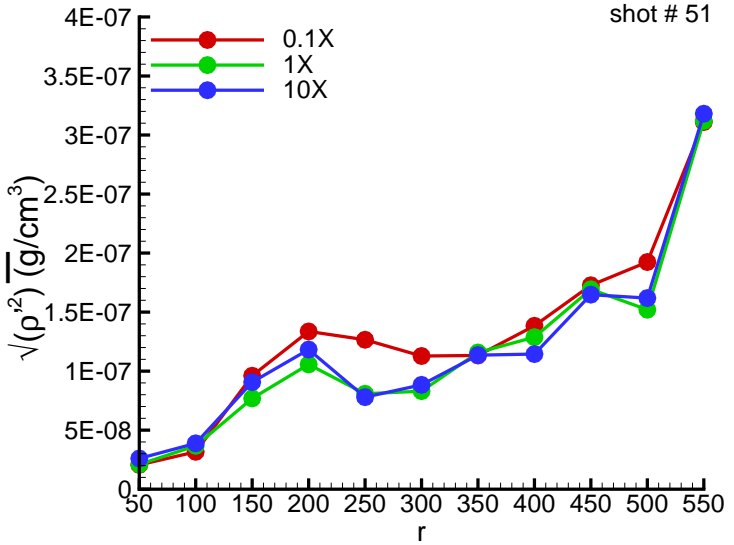

(b)

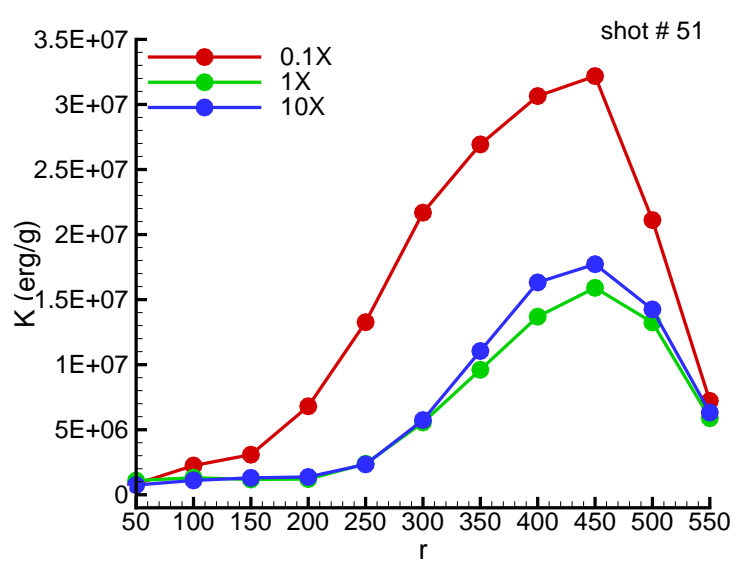

(c)

Figure 15: Distribution of averaged density(a), density fluctuation (b) and turbulence kinetic energy on the spherical shells at varying radius shortly before the $51^{\text {st }}$ shot.

Now, we investigate the density and velocity spherical spectra inside the fusion chamber to understand the turbulence characteristics. Density values are extracted from simulations on uniformly spaced spherical grids at varying radii, and a spherical harmonic decomposition is performed to determine the power spectrum on each surface. Data is compared for all three cases just before the $51^{\text {st }}$ shot. Figure 16 (a), (b) and (c) show the density power spectrum for the $0.1 \mathrm{X}, 1 \mathrm{X}$ and $10 \mathrm{X}$ simulations, respectively. Due to the phenomena that takes place during and after each shot, we anticipate that there are three regimes of turbulence development in the fusion chamber. First, there is a strongly non-equilibrium and nonuniform turbulent state during the energy deposition phase and subsequent shockwave interactions (and reverberations inside the chamber) that produce active turbulence through Richtmyer-Meshkov and Rayleigh-Taylor instability process. Second, there is 
turbulence introduced in the chamber by the influx of vacuum vessel fluid from the port openings (which happens at a slower convective timescale, as opposed to the shockwave timescale of the first phase). Here, there is a better opportunity for a quasi-equilibrium state to arise; a power spectrum with a slope of $-5 / 3$ is expected for a fully equilibrium turbulent flow. Third and finally, after all sources of energy generation disappeared, there is only turbulence decay possible. This will manifest itself as a dissipative process that will show in the power spectra as a steepening of the spectra at the high wavenumber range. Since figure 16 shows spectra at the end of the shot (just prior to a new shot), we observe the flow precisely in the third state of development, i.e., a combined equilibrium spectra with slope close to $-5 / 3$ in the center of the wavenumber range, followed by a much stronger decay at high wavenumbers. The spectra across the different radii shows almost identical decay rates across the range of wave numbers. The spectra is collapsed in most compact fashion for the $0.1 \mathrm{X}$ simulation indicating most amount of energy distribution into smaller scales, but the differences are small between simulations. These spectra of density highlights the mixing state of the gas mixture inside the fusion chamber. The complementary information about the actual state of the turbulence, i.e., the velocity field is shown in figure 17. In these figures we do not observe the intermediate range of $-5 / 3$ but simply the faster decay rate dominated by diffusion. This is consistent with our previous observations regarding the turbulence state in the chamber. At the current energy deposition levels and rate (shot frequency), there is sufficient time after the blast wave to allow conditions in the chamber to quiet down somewhat before the time of the next shot. This explains why the velocity spectra shows a strong "diffusive" decay rate. The same cannot be said about density, because although density is not strictly speaking a passive scalar in compressible flow, it acts nearly as such. A simple thought experiment reveals that it is possible to observe a non-uniform density distribution (and therefore a non-zero spectra) even in the absence of flow. When two fluids are mixed by an external actuation, the fluids will start to mix but if the driving force that moves the fluids stops before the fluids have completely mixed, we will observe a mixture of pure and partially mixed fluids after the flow has stopped completely. Of course, this will not persist indefinitely. The two fluids will eventually mix due to molecular diffusion but on time-scale that is much larger than that induced by the turbulent state. In our context, the density spectra will look exactly like those of the velocity if we wait for a sufficiently long time (and do not introduce another blast wave, of course). 


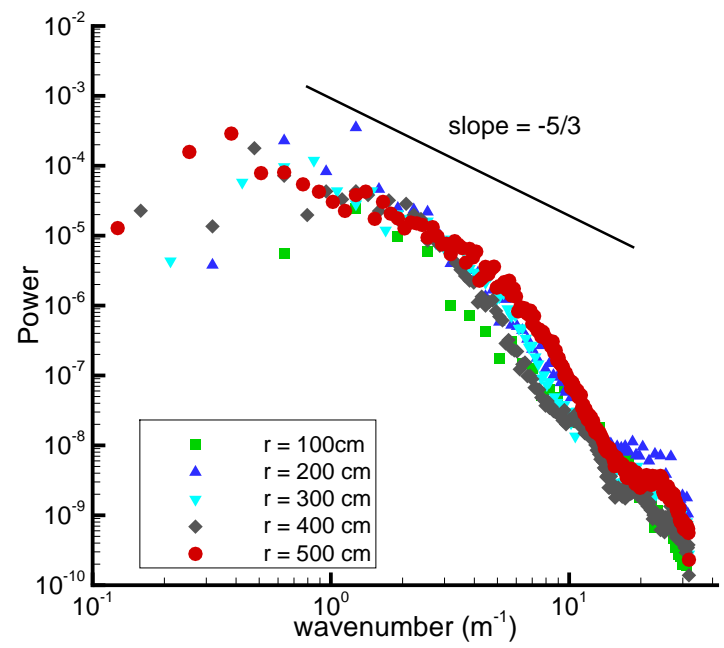

(a) $0.1 \mathrm{X}$

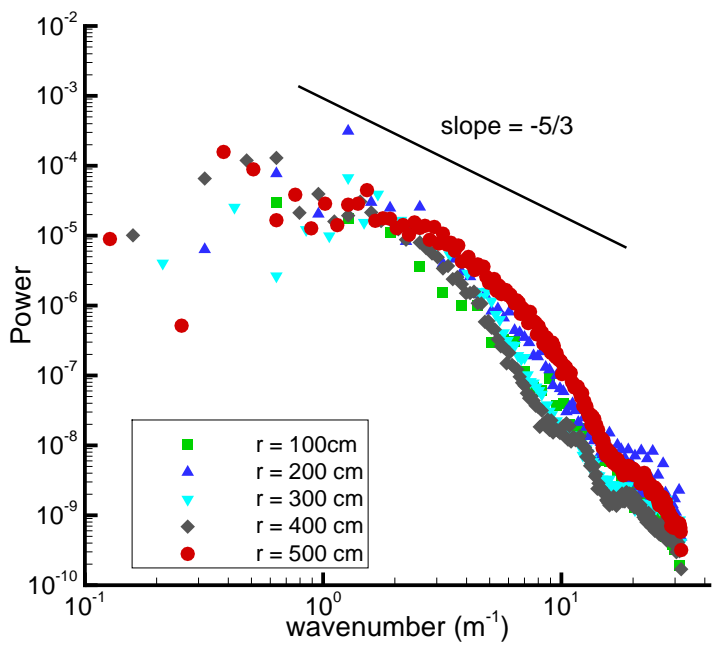

(b) $1 \mathrm{X}$

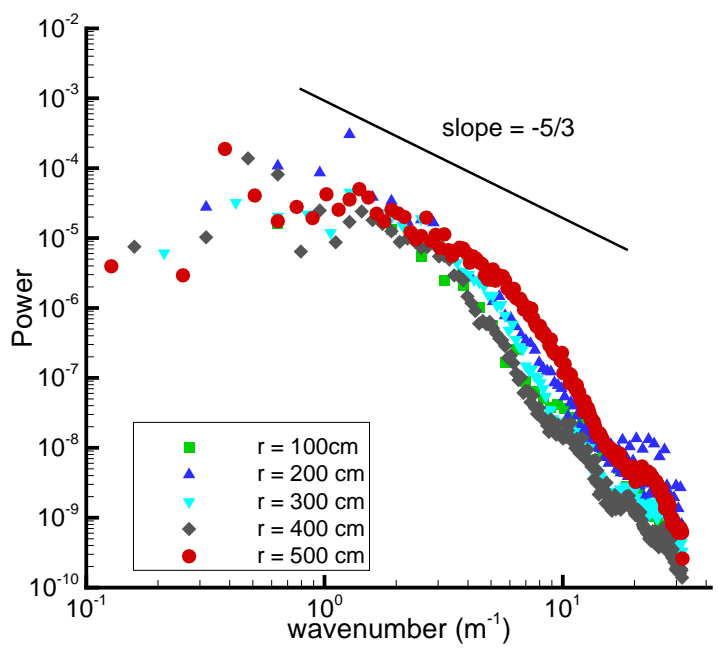

(c) $10 \mathrm{X}$

Figure 16: Density spectra on spheres of varying radius inside the chamber shortly before the $51^{\text {st }}$ shot for the $0.1 \mathrm{X}(\mathrm{a}), 1 \mathrm{X}(\mathrm{b})$ and 10X (c) simulations, respectively. 


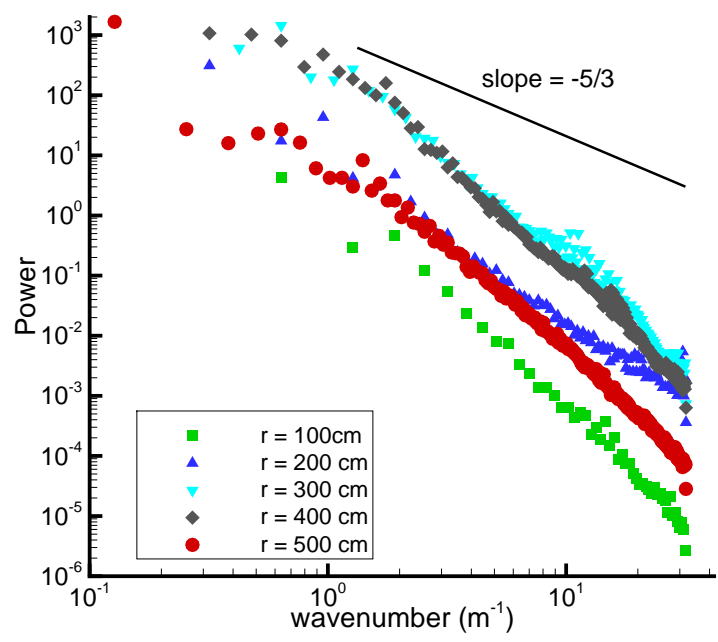

(a) $0.1 \mathrm{X}$

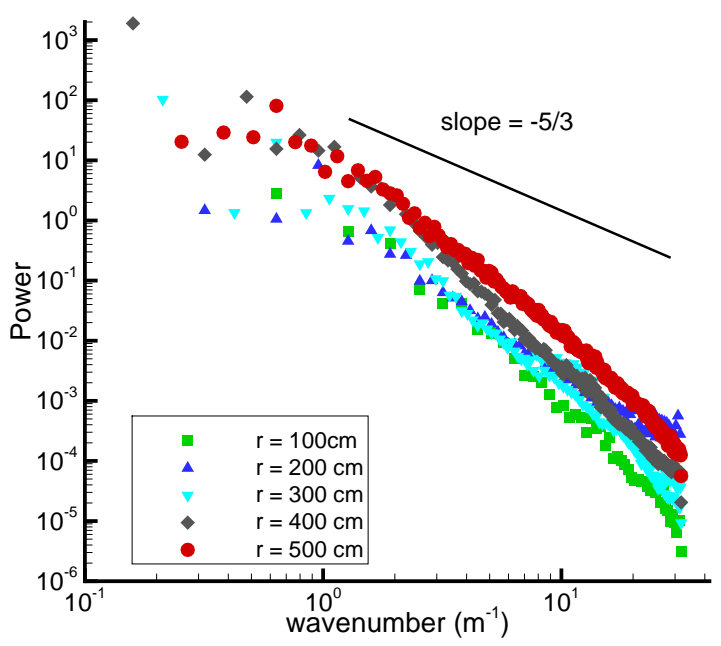

(b) $1 \mathrm{X}$

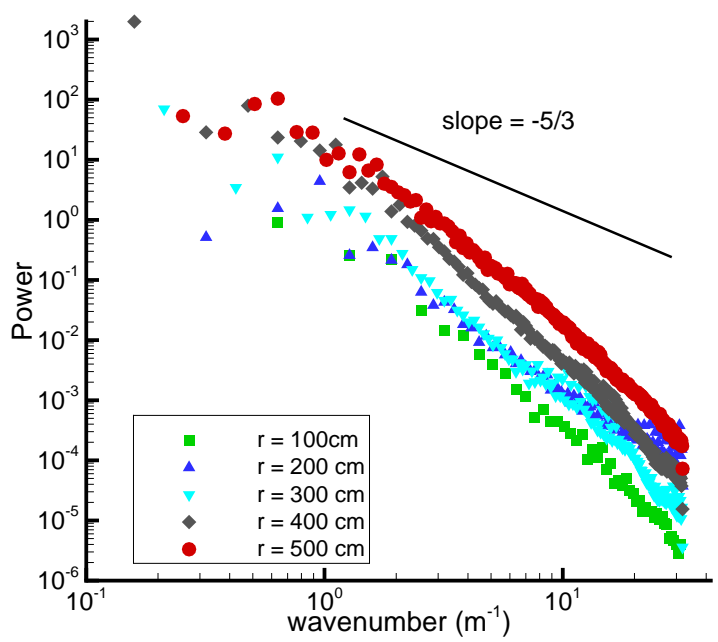

(c) $10 \mathrm{X}$

Figure 17: Velocity spectra on spheres of varying radius inside the chamber shortly before the $51^{\text {st }}$ shot for the $0.1 \mathrm{X}(\mathrm{a}), 1 \mathrm{X}(\mathrm{b})$ and 10X (c) simulations, respectively. 


\subsection{Temperature distribution on the fusion chamber walls}

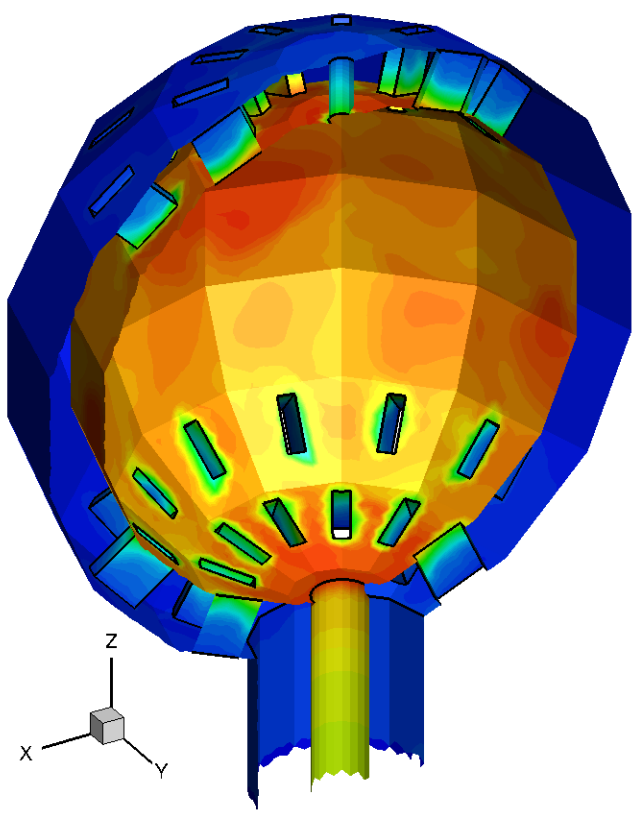

(a) $0.1 \mathrm{X}$

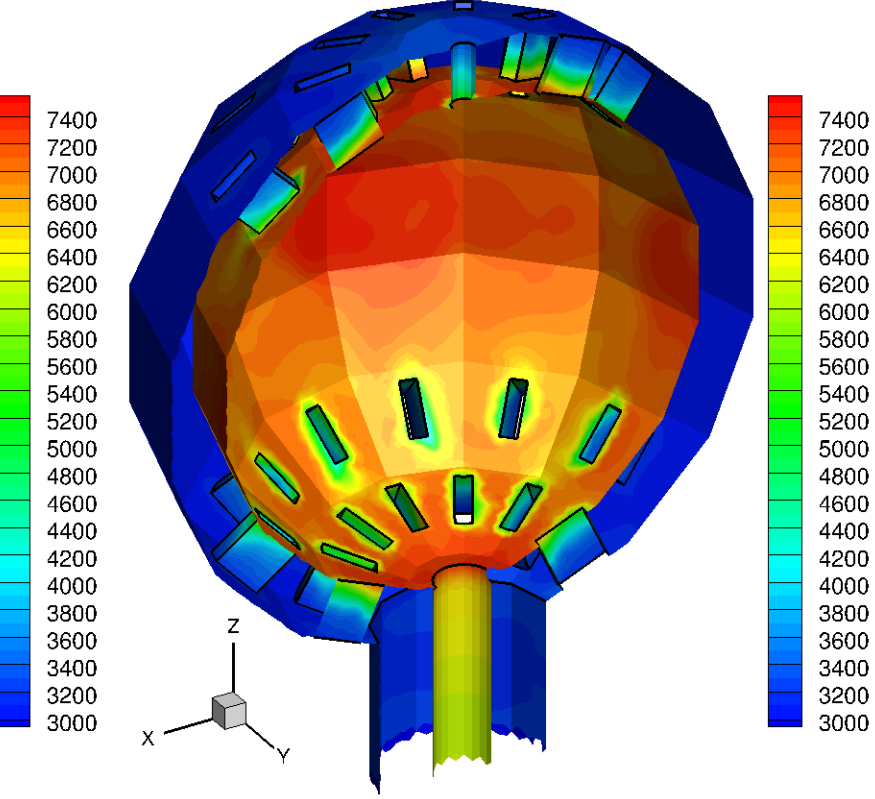

(b) $1 \mathrm{X}$

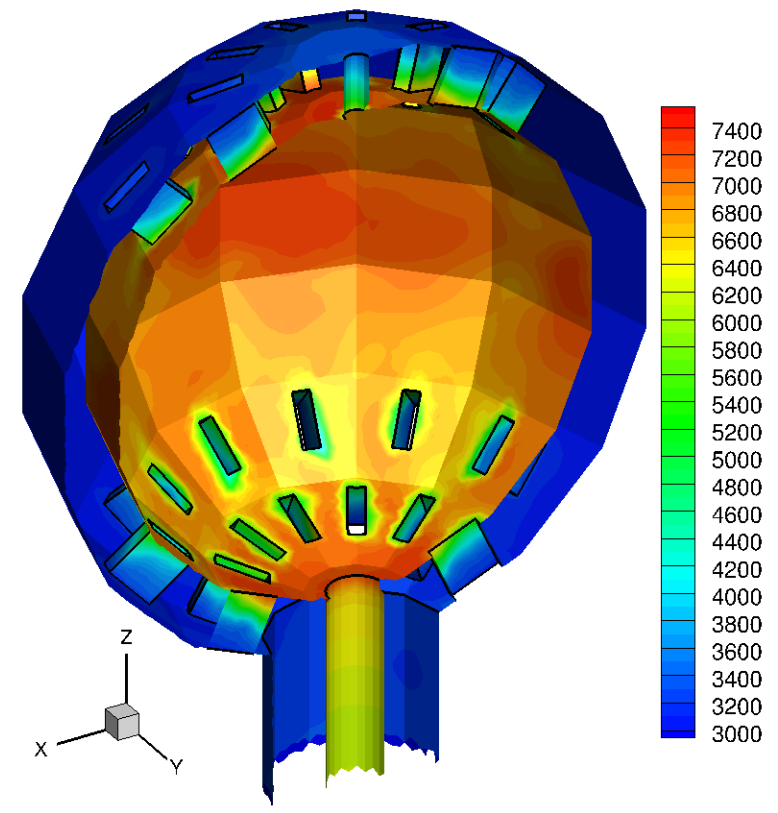

(c) $10 \mathrm{X}$

Figure 18: Temperature contours on the inner chamber wall shortly before the $51^{s t}$ shot.

Figure 18 shows the distribution of the temperature on the walls of the fusion chamber before the $51^{\text {st }}$ shot for all simulations. While there are small differences between cases, overall we observe that the peak temperature happens around the equatorial region of the chamber interior, peak temperatures of $7400 \mathrm{~K}$, and much lower temperatures around the optical beam port regions. There, 
the temperature drops to around $4500 \mathrm{~K}$. These lower temperature regions are the result of the mixing of the chamber gas with the entrained cold gas from the vacuum vessel. Certainly, the temperature distribution changes depending on the time of the cycle at which the data is obtained, but given the approximations made as part of the handshake procedure, to reconcile the more physically accurate description of the radiative hydrodynamics, it is expected that there will be much larger uncertainties on the temperature distributions early in the cycle than later, where the flow physics of dominated by regular hydrodynamics. Furthermore, the temperatures shown in figure 18 must be seen as the temperature distribution in the outer region of a thin boundary layer residing over the fusion chamber wall. This thermal boundary layer (which is not simulated here) must smoothly resolve the transition between the chamber interior conditions and the chamber wall temperature or heat transfer rate, which cannot be known unless the heat-exchange details of the wall ducts, and geometry, is incorporated. 


\subsection{Optical beam pathway data}

In this section, we analyze several quantities of importance along the direction of the laser beam pathway. The fusion chamber possess two groups of ports on each hemisphere (top and bottom). Every group of ports is aligned at the same angle with respect to the vertical and distributed uniformly in the azimuthal direction, along 12 segments, as can be seen in figure 1. The angles of the group of ports is $\pm 25^{\circ}$ and $\pm 47.5^{\circ}$ with respect to the vertical $z$ axis, with the positive angles denoting the top ports. Figure 19 shows highlighted rendering of the line of sight volume covered by the optical port openings inside the chamber (these volumes extend outwards into the vacuum vessel as well, not shown).

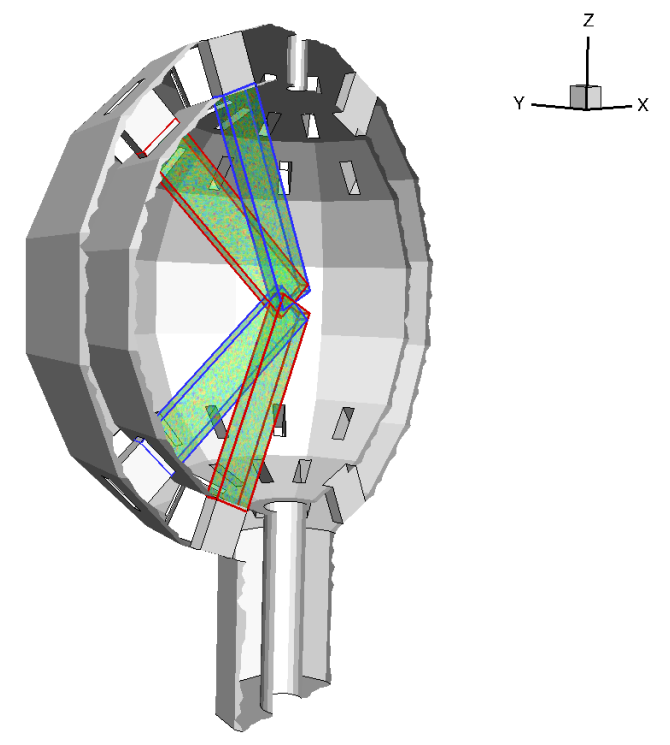

Figure 19: Extracted (selected) volume regions for histogram plots with pseudo-data for representation purpose.

Figure 20 shows density profiles along the center of the optical beam pathway from the center of the fusion chamber to the outer ports in the vacuum vessel for the four different types of ports, at one of the 12 segments of the chamber wall. Note that the density profiles are very similar for all simulations and those for $\pm z \measuredangle 25^{\circ}$ are indistinguishable from the profiles along the vertical axis, shown in figure 13 . The profiles along $\pm z \measuredangle 47.5^{\circ}$ show a slight difference outside of the fusion chamber with an overshot of the density there before dropping to the same reference value of the conditions in the vacuum vessel. Figure 21 shows histograms (discrete approximations to probability density functions) of the density at different radii from the fusion chamber center obtained from the density within the optical path volume for simulation 1X. These plots highlight the variability of the density within the beam volume and assess to degree of variability of the density in that region. Note that the density variance, the width of the histograms, is not large, of the order of $10^{-7} \mathrm{~g} / \mathrm{cm}^{3}$, which is similar to the spherical shell-averaged variance shown in figure $15(\mathrm{~b})$.

Figure 22 shows all the histograms for the four representative optical pathway volumes as a function of radius for simulation 1X. Similarly, figure 23 shows a comparative matrix of histograms for the three simulations a the single $+z \measuredangle 25^{\circ}$ direction. The simulation results are very similar all cases. Finally, figure 24 shows the pressure measured on the vacuum vessel top and bottom walls just at the center of the port openings as a function of time for all three simulations (which are indistinguishable). As can be seen from the figure, the mean pressure is approximately 23,500 


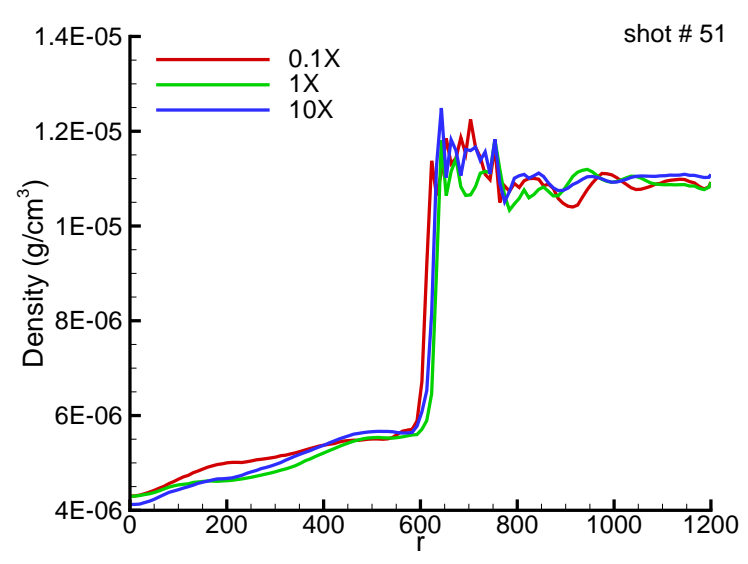

(a) $+z \measuredangle 25^{\circ}$

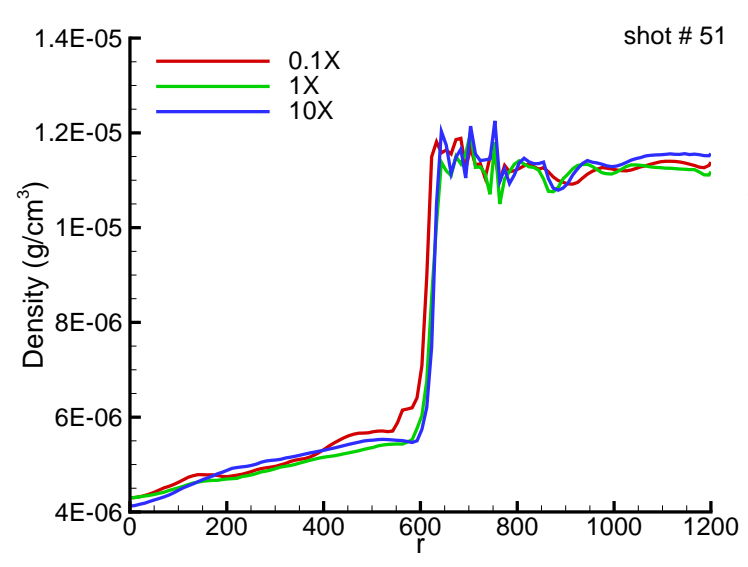

(c) $-z<25^{\circ}$

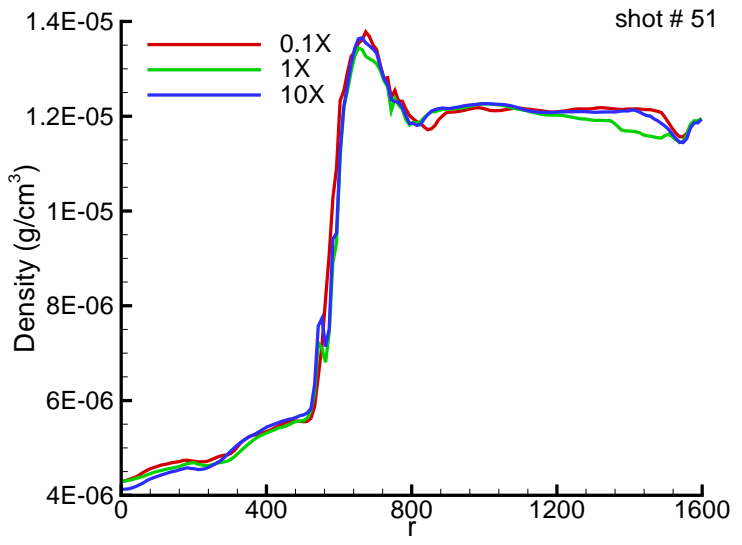

(b) $+z \measuredangle 47.5^{\circ}$

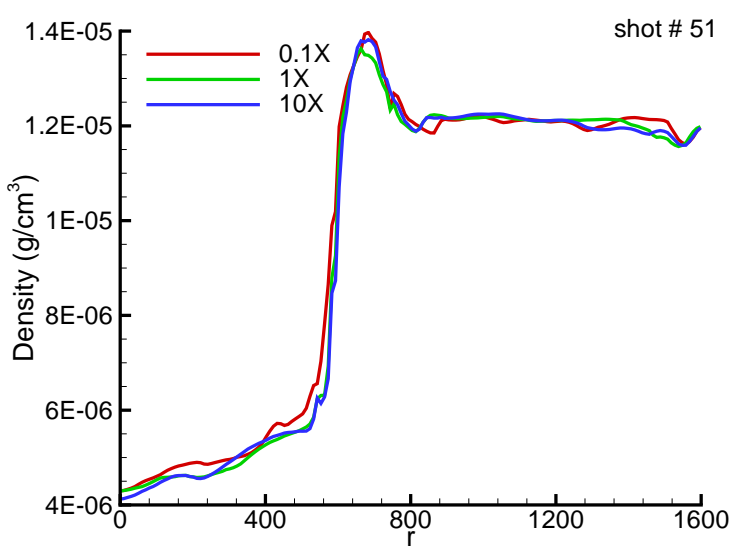

(d) $-z<47.5^{\circ}$

Figure 20: Density profiles along the line passing through 4 different ports at one of the 12 segments of the fusion chamber shortly before the $51^{\text {st }}$ shot.

dyne $/ \mathrm{cm}^{2}$, the peak pressure fluctuation intensity is of the order of $1000 \mathrm{dyne} / \mathrm{cm}^{2}$ for the $\pm z \measuredangle 25^{\circ}$ and 500 dyne $/ \mathrm{cm}^{2}$ for the $\pm z \measuredangle 47.5^{\circ}$ ports. There is no appreciable difference between the top and bottom ports. 

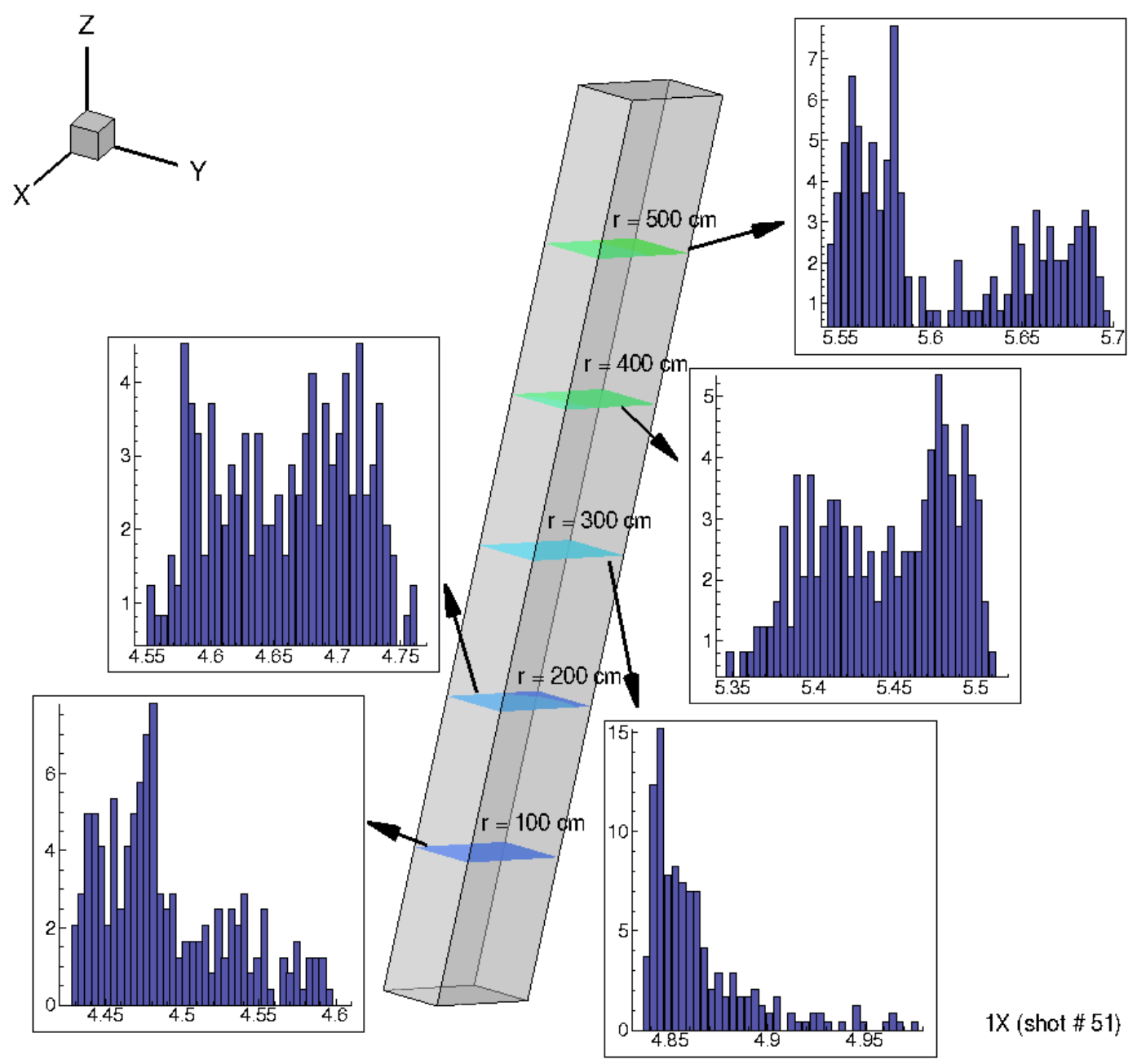

$1 \mathrm{X}$ (shot \# 51)

Figure 21: Composite representation of the optical pathway volume and several density histograms along the $+z \measuredangle 25^{\circ}$ port direction. (Density measured in $\mu \mathrm{g} / \mathrm{cm}^{3}$.) 


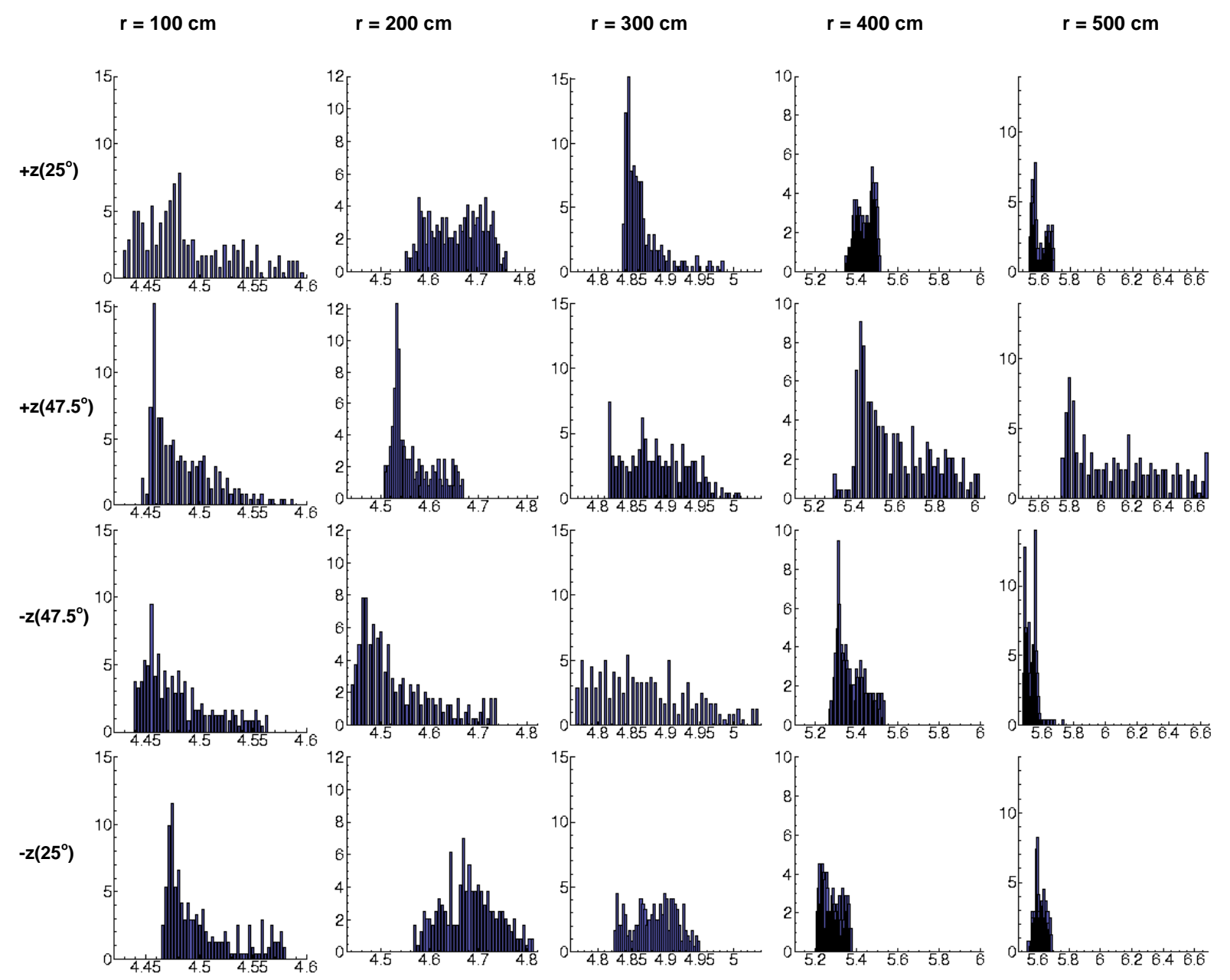

Figure 22: Density histograms varying with radial distance inside 4 different volume zones from the center of the fusion chamber to the chamber ports at one of the 12 geometric segments for the 1X simulation. (Density measured in $\mu \mathrm{g} / \mathrm{cm}^{3}$.) 


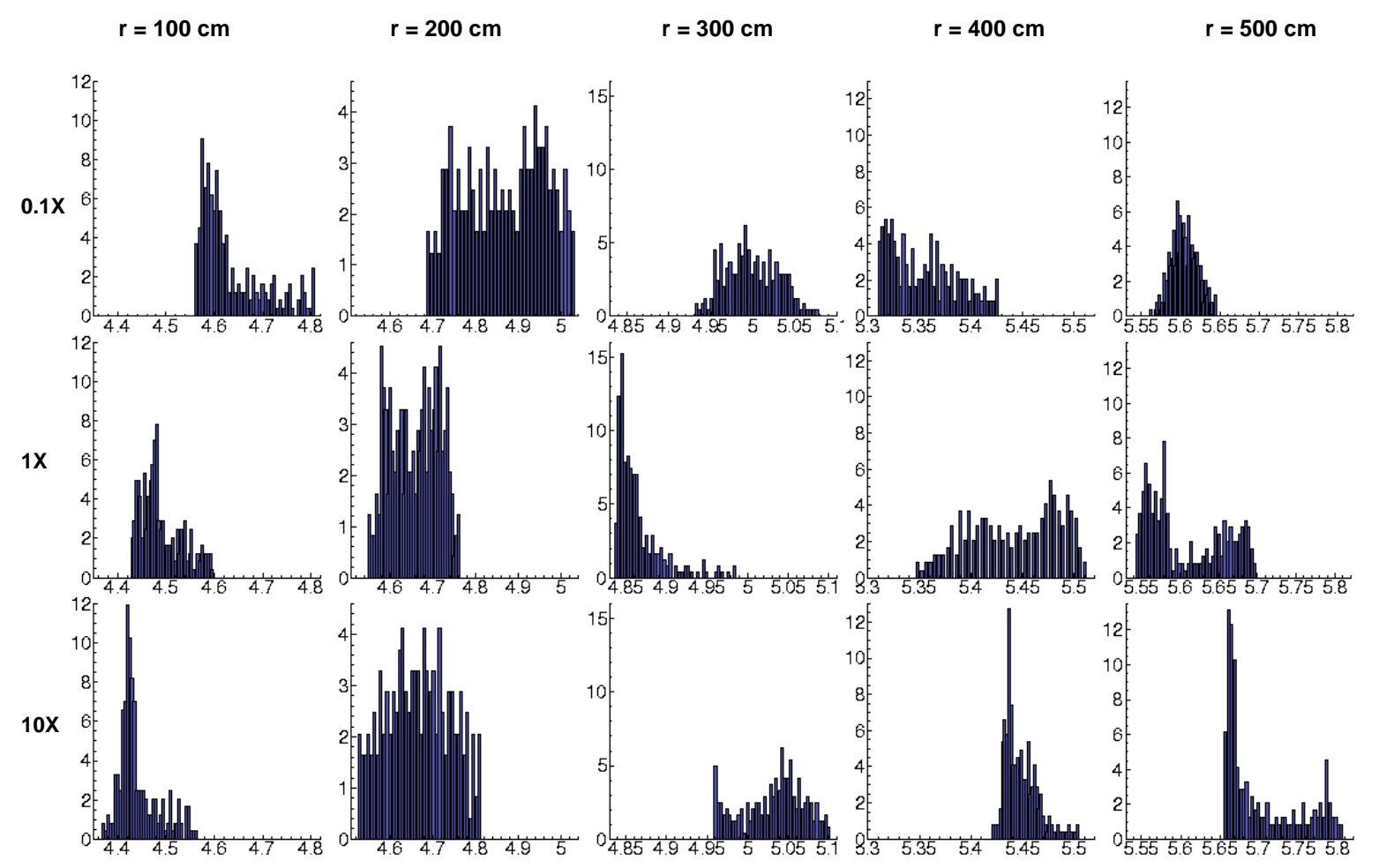

Figure 23: Density histogram varying with radial distance inside a volume zone from the center of the fusion chamber to one of the top chamber ports at $+z \measuredangle 25^{\circ}$ with the vertical axis for $0.1 \mathrm{X}, 1 \mathrm{X}$ and 10X cases (top to bottom). 


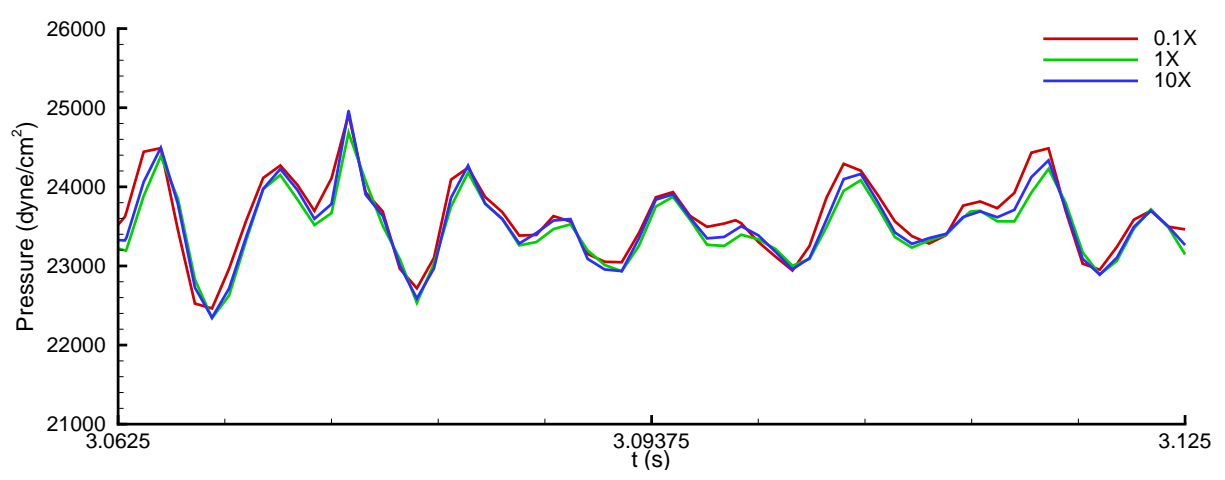

(a) $+z<25^{\circ}$

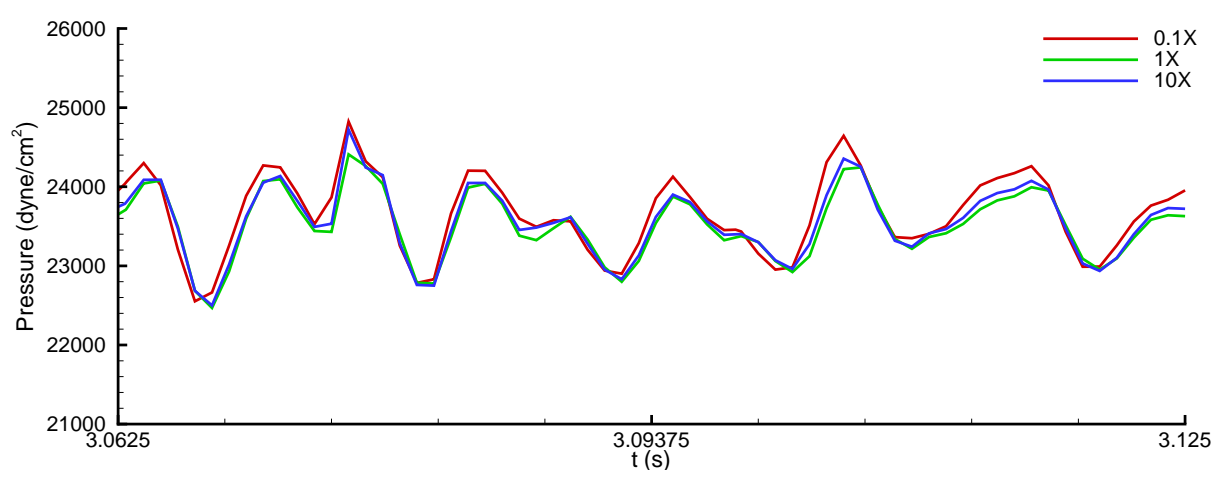

(b) $-z \measuredangle 25^{\circ}$

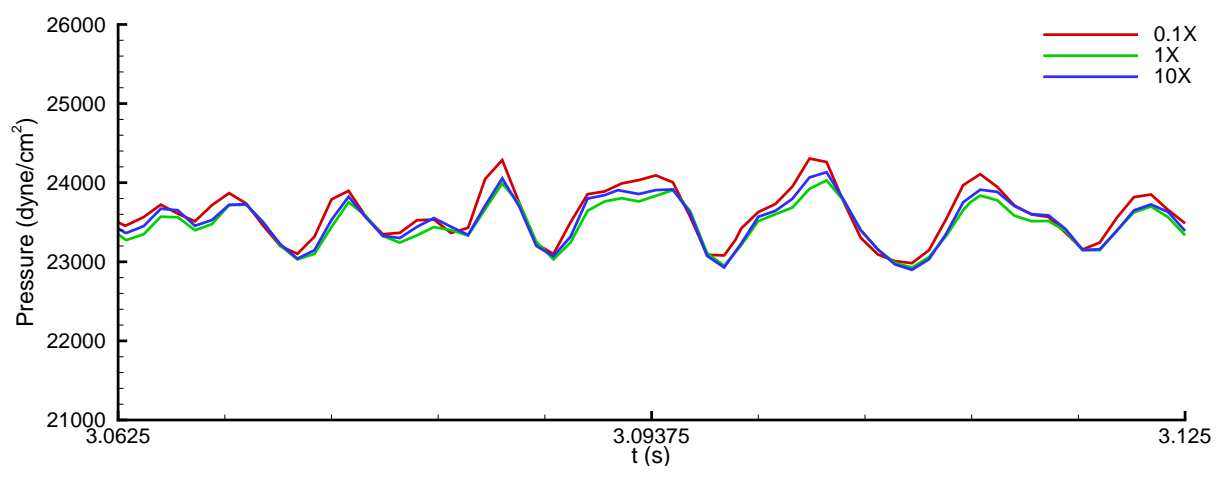

(c) $+z \measuredangle 47.5^{\circ}$

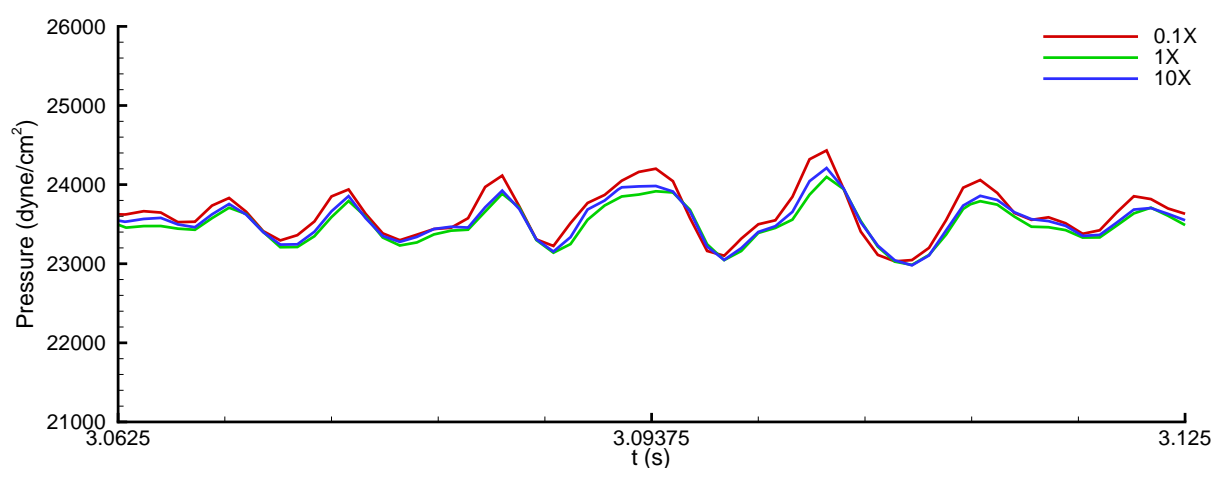

(d) $-z \measuredangle 47.5^{\circ}$

Figure 24: Pressure on the vacuum vessel top and bottom walls as a function of time between shots 50 and 51. 


\section{Conclusions and Perspective for Future Improvements}

The present report provides a synopsis of the simulation results and the sensitivity of the results to the energy deposition approximations employed in the study. Overall, within the range of parameters explored, there is only modest change of the hydrodynamics to the details of the blast model inferred from the BUCKY simulations. Most quantities of interest discussed above were not sensitive to the differences in the simulations. The main observable difference appears in simulation $0.1 \mathrm{X}$ where it is clear that the level of turbulence intensity increased in comparison with the other simulations. Despite this difference, and as it pertains to the laser beam or target flight environment, conditions quiet down sufficiently after each shot for the turbulence to die down before a new shot is induced. This is a particular regime for the shot frequency employed in the simulations and the conclusions will still hold if the frequency is lowered further. It is unclear how the environment will change if the frequency were to be increased, such that the turbulence environment will remain highly energetic at the time of a new shot. In such conditions, it might be possible for the fusion chamber fluid to maintain a higher degree of turbulence. On one hand, this might be problematic for the target flight but, on the other hand, it might be beneficial for the laser beam targeting and focussing because higher turbulence intensities will enhance mixing and result in more uniform density conditions in the chamber.

In terms of future improvements, we envision three enhancement levels. One is the careful assessment of resolution on the present results with the current computational infrastructure. This is tentatively underway but the conclusion depends on availability of computational resources. The second enhancement would be the use of more accurate gridding technology such as fully unstructured or block-structured overlapping (Chimera) meshing approach that would allow resolution of the thermal (and possibly mechanical) boundary layers around the fusion chamber, as well as other secondary flow regions that are currently marginally resolved. This will help answer some of the thermodynamic questions about phase changes of the debris material as it reaches the fusion chamber walls, which are supposed to be colder than the interior gas. Certainly, this will result in more expensive simulations and investment in development in a new simulation code or using an alternative code. Finally, the third enhancement will certainly necessitate the integration of the radiation hydrodynamics as well as target geometry to fully model the energy deposition in the fusion chamber gas. At the same time, one will require the modeling of the heat transfer elements that constitute the walls of the chamber (which are not really composed of a single solid block, as modeled in this study, but are made of a complex heat exchange system). This last enhancement will enable us to achieve detailed energetic balance and presumably allow a truly $\mathrm{n}^{\text {th }}$ shot state to be achieved. In addition, other questions about the details of the hydrodynamic transient of the fusion from cold startup, may be answered. It is unnecessary to state it, but we do anyway, that the computational cost of these last type of simulation will require tremendous development and

computational resources. This will only be warranted in the very last stages of development where one or two definitive chamber geometries have been selected for possible test trials. 


\section{References}

[1] C. Pantano, R. Kramer, and H. Uddin. CFD simulation in support of the dynamic chamber systems strategic initiative. Technical report, University of Illinois at Urbana-Champaign, 2012.

[2] C. Pantano and H. Uddin. VTF-BUCKY handshake update version 2. Technical report, University of Illinois at Urbana-Champaign, 2012.

[3] G.B. Whitham. Linear and Nonlinear Waves. Wiley, 1999. 